\title{
Alternative Positron-Target Design for Electron-Positron Colliders
}

\author{
Richard J. Donahue and W. R. Nelson \\ Environment, Health and Safety Division \\ Lawrence Berkeley Laboratory \\ University of California \\ Berkeley, California
}

and

\section{Environment,Safety and Health Division}

Stanford Linear Accelerator Center

Stanford University

Stanford, California 94309

This report has been reproduced directly from the best available copy.

This work was supported by the Director, Office of Energy Research of the

U. S. Department of Energy under Contract No. DE-AC03-76SF00098 


\title{
Alternative Positron-Target Design for Electron-Positron Colliders*
}

\author{
Richard J. Donahue $\dagger$ \\ Lawrence Berkeley Laboratory * \\ University of California \\ W. R. Nelson \\ Stanford Linear Accelerator Center $\ddagger$ \\ Stanford University
}

\begin{abstract}
Current electron-positron linear colliders are limited in luminosity by the number of positrons which can be generated from targets presently used. This paper examines the possibility of using an alternate wire-target geometry for the production of positrons via an electron-induced electromagnetic cascade shower.
\end{abstract}

\footnotetext{
*Work supported by the Department of Energy, contract DE-AC03-76SF00515

$\dagger$ Partial fulfillment for M. S. Degree at University of Lowell

$\ddagger$ Work supported by the Department of Energy, contract DE-AC03-76SF00098
} 


\section{Introduction}

One of the major design problems which must be overcome for the next generation of electron-positron colliders is a source of positrons which will meet the projected luminosity $\left(\mathrm{cm}^{-2}\right)$ requirements ${ }^{[1]}$. These luminosity requirements are at least a factor of one hundred higher than for present-day $\mathrm{e}^{+} \mathrm{e}^{-}$colliders. One method to increase luminosity is to reduce the cross-sectional area $\left(\sigma_{x} \times \sigma_{y}\right)$ of the colliding beams. The other way is to increase the beam current. The $\mathrm{e}^{+}$target currently used at the Stanford Linear Collider (SLC) is approaching its design limitation, so there is considerable incentive to find new ways to produce the needed number of positrons ${ }^{[2]}$. This paper explores the idea of using a thin cylinder, or wire, to produce positrons as an alternative to the semi-infinite geometry now used.

\section{Properties of the Electromagnetic Cascade Shower}

\subsection{General}

When charged particles are transported through a material, they lose energy through collisions and radiation processes. Collision processes result from excitation and/or ionization of atoms in the material. Energy is lost through secondary electron emission and is deposited locally. This process accounts for the majority of the heat deposition in targets. Energy loss by radiation processes (i.e., bremsstrahlung) is distributed among secondary particles whose energy may reach the energy of the incident electron. At high incident electron energies this is the dominant energy-loss mechanism. The dominant photon cross section at high energies is pair production, or materialization, whereby an electron-positron pair is created. 'The electron-positron pairs can also radiate bremsstrahlung photons. 'This sequence continues, with the energy of each newly created particle decreasing with each interaction, resulting in what is called an electromagnetic (EM) cascade shower. 


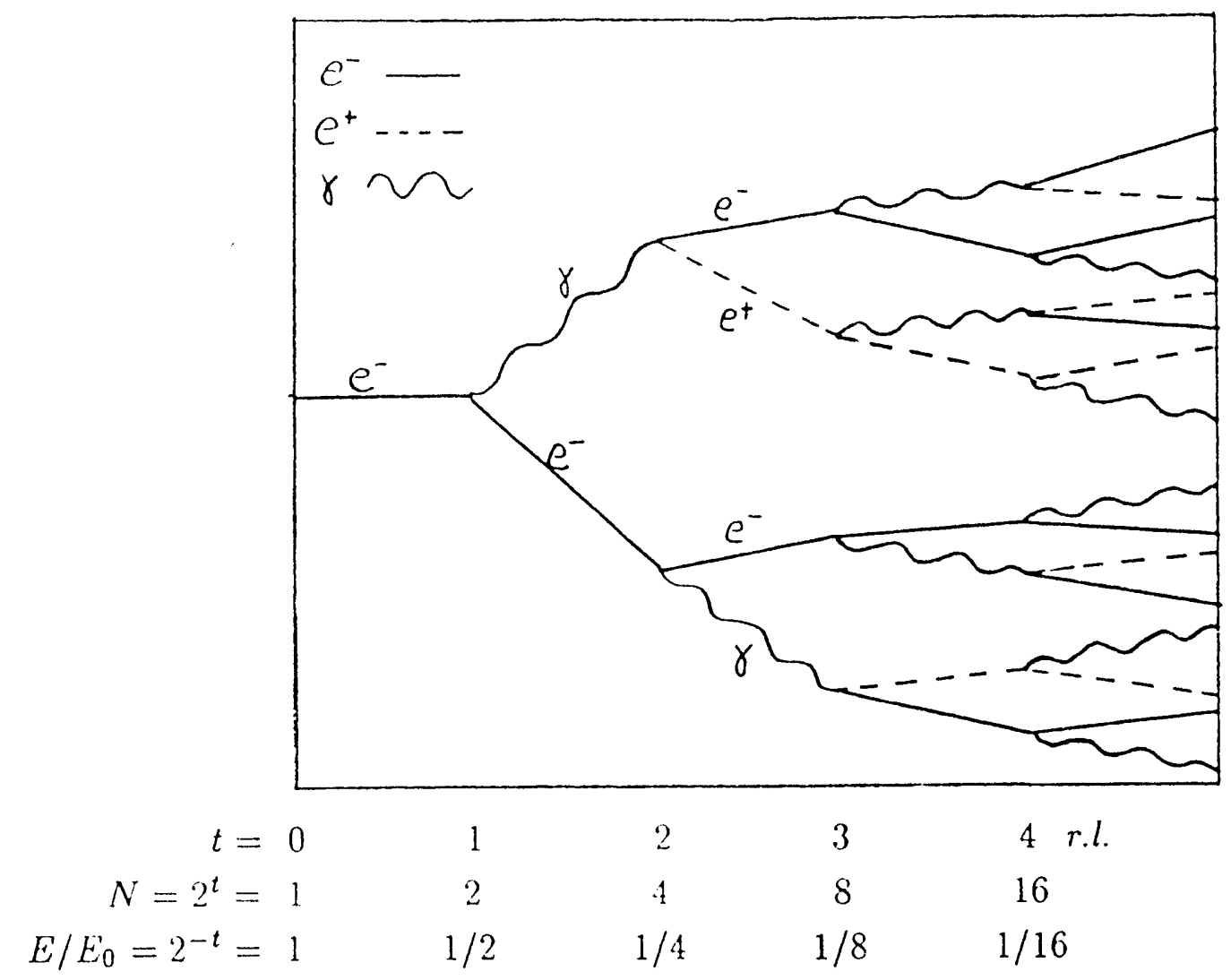

Fig. 1. Simplified schematic of the initial portion of an EM cascade shower.

Figure 1 is a simplified schematic of the initial portion of a high-energy shower. The horizontal axis has units of radiation lengths, along side of which the number of secondaries $\left(e^{ \pm}, \gamma\right)$, as well as the energy of each secondary, is indicated. From this diagram one can deduce ${ }^{[3]}$ that the number of secondaries is proportional to the incident energy, $E_{0}$, and inversely proportional to the cutoff energy, $E_{c u t}$. Namely,

$$
N=N_{e^{-}}+N_{e^{+}}+N_{\gamma} \propto \frac{E_{0}}{E_{c u t}} .
$$

Thus, for a given cutoff energy and for high-energy beams, the number of secondaries scales as the nergy of the incident beam. Fig. $2 a$ and $2 b$ are EGSgenerated ${ }^{[*]}$ shower simulations initiated by single $1 \mathrm{GeV}$ and $10 \mathrm{GeV}$ electrons, respectively. The tmurien target has a diameter of one centimeter and is 6 radiation lengths (r.l.) Kng $(\approx 2.1-\mathrm{cm})$. (harged particles are shown as solid lines and photons as dotted lines and the intensity of the lines are proportional to the energy. 

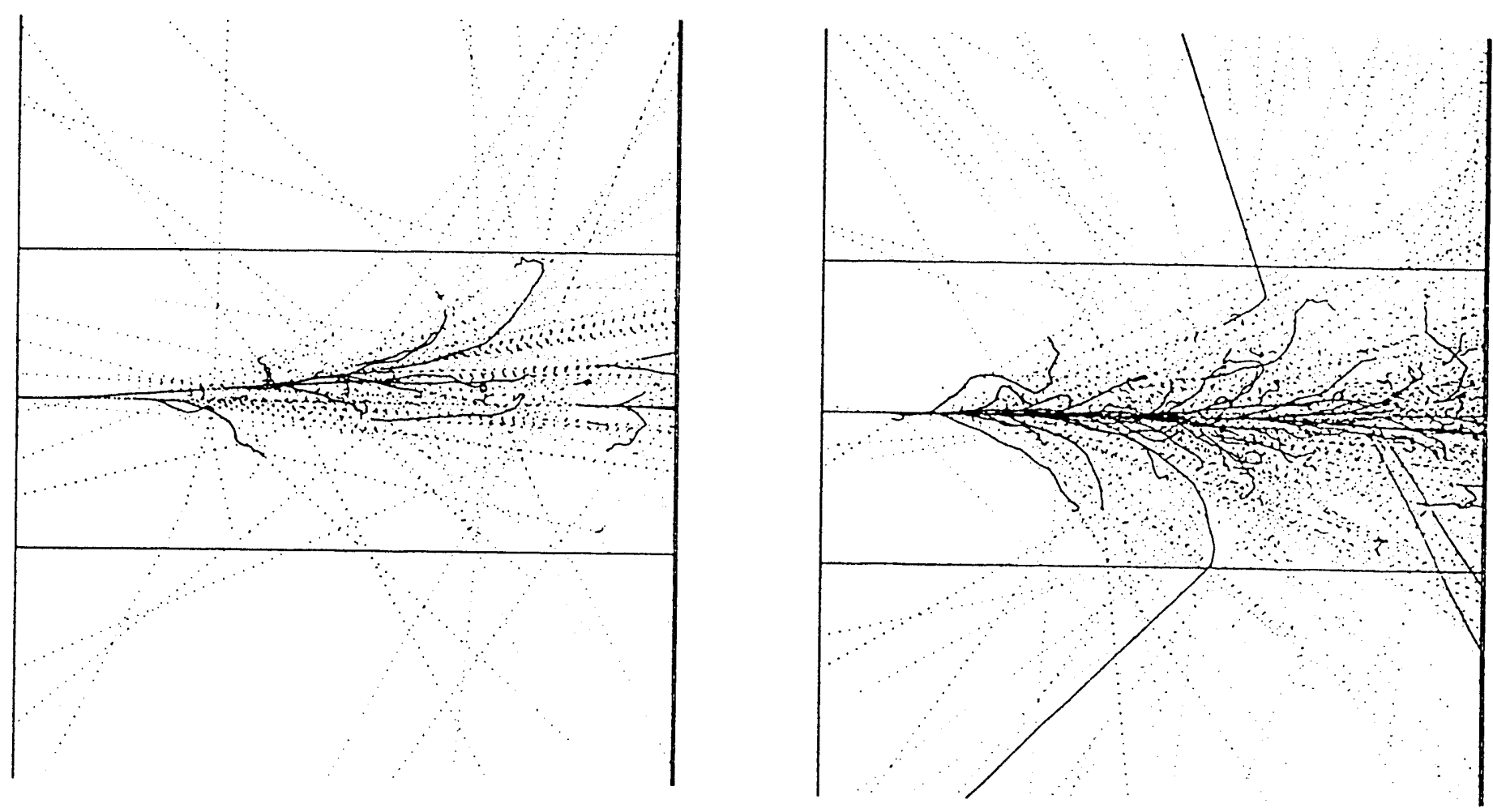

Fig. 2. EGS4 (graphical) simulation ${ }^{(1)}$ of an EM cascade shower: a) $1 \mathrm{GeV}$, b) $10 \mathrm{GeV}$.

A radiation length can be thought of as the distance over which, on the average, the incident electron loses all but a fraction $\frac{1}{e}$ of its energy to bremsstrahlung. This is a convenient unit for scaling longitudinal distances in an EM cascade shower. The radiation length values for various materials have been tabulated by Tsai ${ }^{\left[{ }^{(j)}\right.}$, whose formula can be approximated by ${ }^{[6]}$

$$
X_{0}=\frac{716.4 A}{Z(Z+1) \ln (287 / \sqrt{Z})}\left(\mathrm{g} / \mathrm{cm}^{2}\right) \text {, }
$$

where $Z$ is the atomic number and $A$ is the atomic weight of the medium. For tungsten $(Z=74)$ a radiation length corresponds to $0.35-\mathrm{cm}$.

The mean longitudinal shower profile of the energy deposition follows a gamma distribution of the form ${ }^{[n]}$

$$
\frac{d E}{d t}=E_{0} b \frac{(b t)^{a-1} e^{-b t}}{\Gamma^{\prime}(a)}
$$

where $E_{0}$ is the electron energy $(\mathrm{GeV}), t$ is the shower depth (r.l.), and the constants $a$ and $b$ are parameterized in terms of $Z$ and the natural logarithm of $E_{0}$. 
Figure 3 shows a cascade shower profile $e^{[6]}$ generated in iron at $30 \mathrm{GeV}$ using the EGS4 Monte Carlo code ${ }^{[4]}$ with a (total) energy cutoff of $1.5 \mathrm{MeV}$ for both charge particles and photons. The shower maximum in the energy deposition curve (histogram) is seen to occur at about 7-r.l.

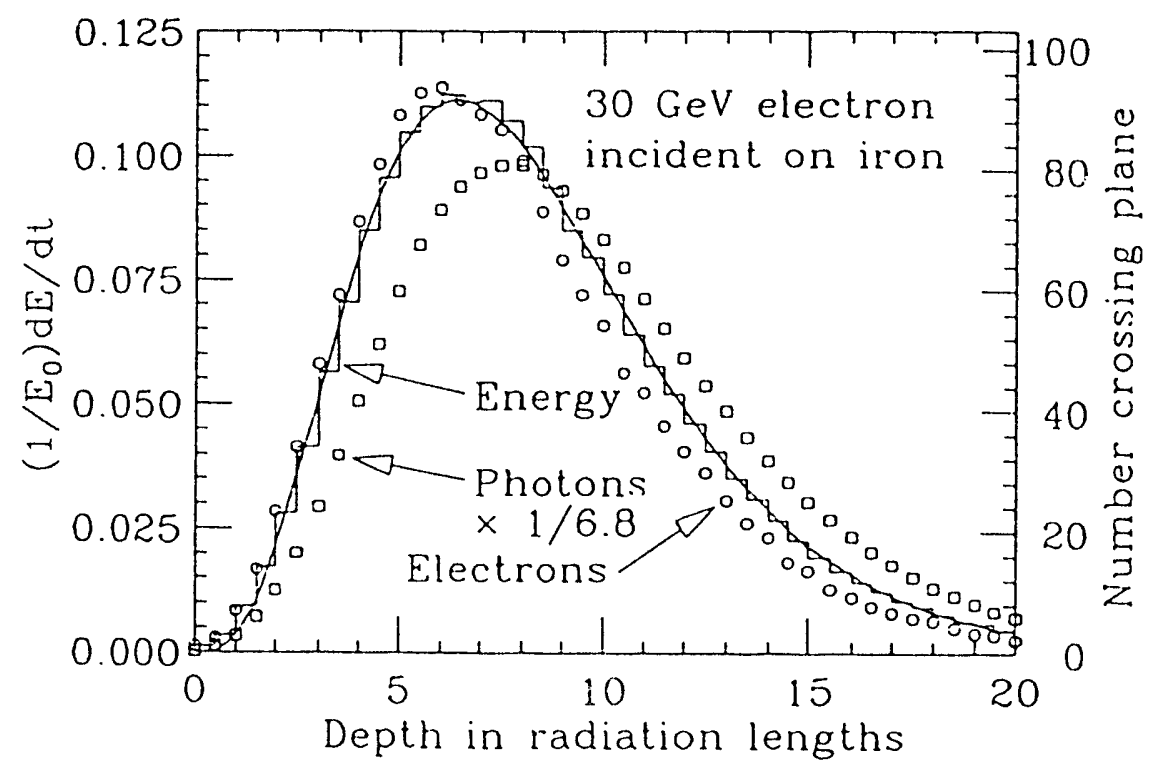

Fig. 3. EGS4 simulation ${ }^{[6]}$ of a $30 \mathrm{GeV}$ shower in iron. The histogram shows fractional energy deposition (per r.l.) and the curve is a gamma-function fit. The corresponding electron and photon numbers are shown as points.

The number of photons is larger than the number of charged particles, showing how quickly the latter convert energy to bremsstrahlung. Since photons interact catastrophically, as opposed to continuously as in the case of low-energy charged particles, much of the energy will escape the target in the form of photons. For a 6 to 7 -r.l. semi-infinite slab of tungsten, about $25 \%$ of an incident $33 \mathrm{GeV}$ beam will be absorbed in the target. The escaping energy produces showers downstream, depositing energy and creating induced radioactivity.

* Shower maximum will also be at $\mathrm{\tau}$-r.l. for our 33 GeV design energy since the longitudinal behavior varies slowly with In $E_{0}$. 
The current SLC target is about one centimeter in diameter and 6-r.l. long. It was designed ${ }^{[8]}$ slightly less than 7 -r.l., since a disproportionately large fraction of the energy is absorbed near shower maximum-i.e., making the target a little shorter reduces the total power absorbed in the target, which in turn decreases both radioactivity and temperature-rise problems.

After briefly discussing the general characteristics of the EM cascade shower, the fundamental interactions which produce the cascade are discussed. These are pair production for photons and bremsstrahlung for charged particles.

\subsection{Photon InTERACTIONS}

Photons interact mainly through three processes: photoelectric effect, Compton scattering, and pair production. Figure 4 shows the cross sections for these processes as a function of photon energy for copper ${ }^{[9]}$.

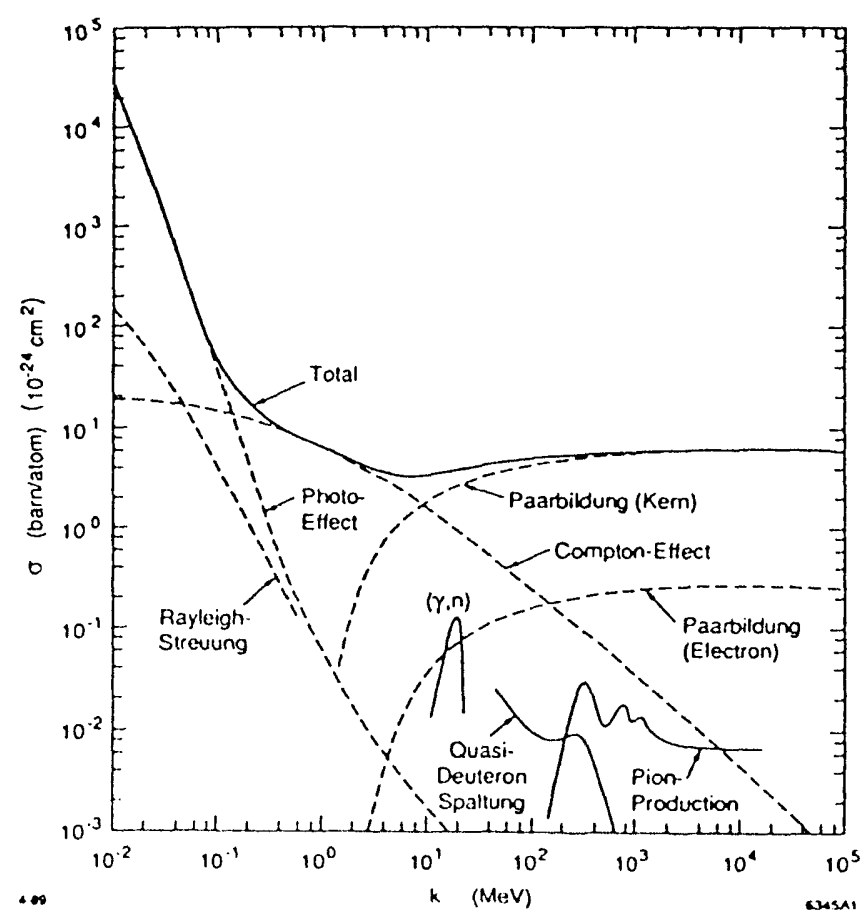

Fig. 4. Photon cross sections for copper ${ }^{|m|}$ 
Note that above about $10 \mathrm{MeV}$, the photonuclear cross sections (giant resonance, quasi-deuteron, pion production) come into play, but these cross sections are down by $1 \frac{1}{2}$ to 3 orders of magnitude.

Figure 5 shows the relative importance of the three dominant reactions ${ }^{[10]}$, with the lines designating where cross sections become equal. This clearly demonstrates that for a high- $Z$ material, such as tungsten $(Z=74)$, pair production begins to dominate when photon energies exceed $5 \mathrm{MeV}$.

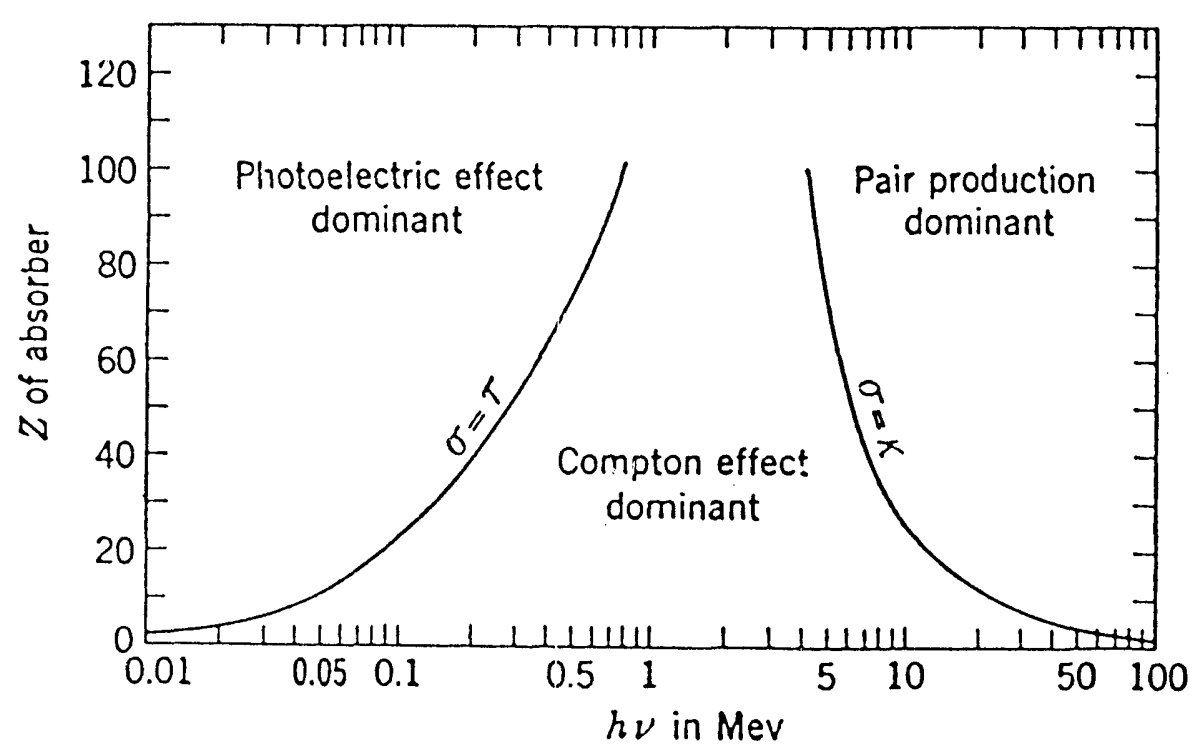

Fig. 5. Comparison of photon cross sections ${ }^{[10]}$.

Pair production is the mechanism whereby a photon is completely abscined and an $\mathrm{e}^{+} \mathrm{e}^{-}$pair is produced. This is also known as materialization since all the photon energy is transformed into matter and kinetic energy. The threshold for this reaction is twice the electron rest mass energy, $2 m_{0} c^{2}(1.022 \mathrm{MeV})$. 
Figure 6 shows the energy distribution of the pairs ${ }^{[10]}$. The abscissa is the fraction of the total kinetic energy of the $\mathrm{e}^{+} \mathrm{e}^{-}$pair which is received by the positron. This shows that, as the photon energy increases, the available kinetic energy tends to get shared more equally among the two charged particles.

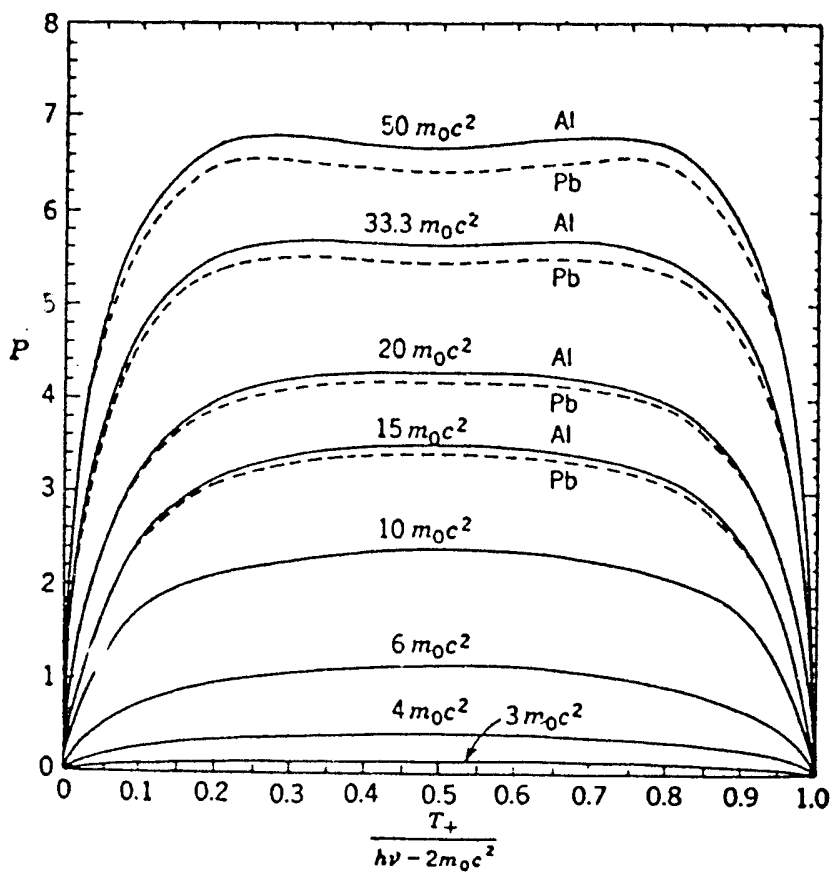

Fig. 6. Energy distribution of $\mathrm{e}^{+} \mathrm{e}^{-}$pairs ${ }^{[10]}$.

The characteristic angle ${ }^{\star}$ between the incident photon of kinetic energy $k$ (or $h \nu$ ) and the created pairs is given by

$$
\theta_{\text {pair }}=\frac{m_{0} c^{2}}{k}
$$

For example, for $k \geq 100 \mathrm{MeV}, \theta_{\text {pair }} \leq 0.3^{\circ}$, which explains why $\mathrm{e}^{+} \mathrm{e}^{-}$pairs are essentially forward-produced, particularly at high photon energies.

* Both the bremsstrahlung and pair production cross sections are scaled in terms of a char. acteristic angle, which is the electron rest-mass energy divided by the incoming (total) energy. 
The attenuation coefficient (i.e., cross section) for pair production varies as

$$
\kappa \simeq Z^{2} \ln k
$$

for low values of $k$, and as

$$
\kappa \simeq Z^{2} \frac{7}{9 X_{0}}
$$

for high energies, where $X_{0}$ is the radiation length of the material. Pair production is induced by the strong electric field surrounding the nucleus. At large distances from the nucleus the electric field is screened by the orbital electrons, and this screening becomes important for high- $Z$ materials and high energies.

The theory of pair production is closely related to bremsstrahlung and the equations describing the two processes are very similar. In bremsstrahlung, an electron makes a transition between two positive energy states and a photon is emitted instead of being absorbed as in pair production. This is illustrated in the Feynman diagrams in Fig. 7.

Pair Production

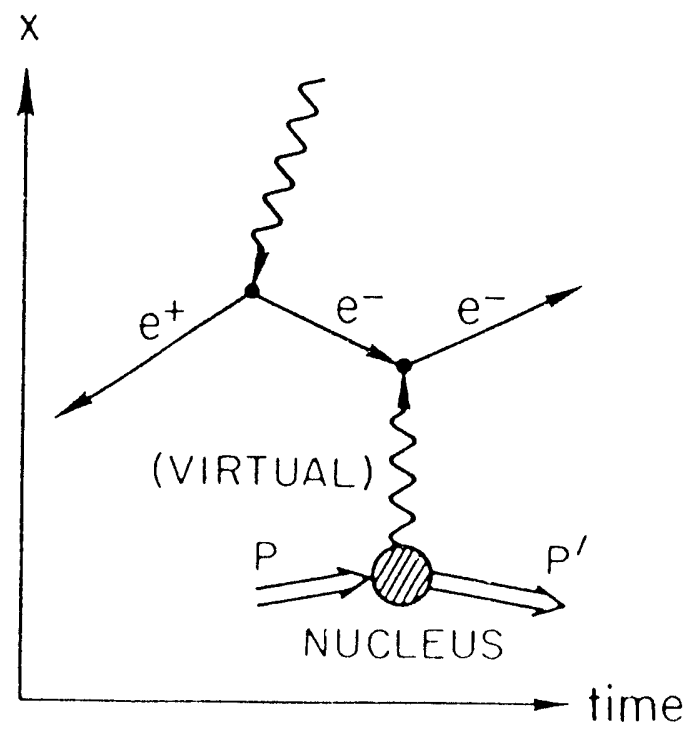

Bremsstrahlung

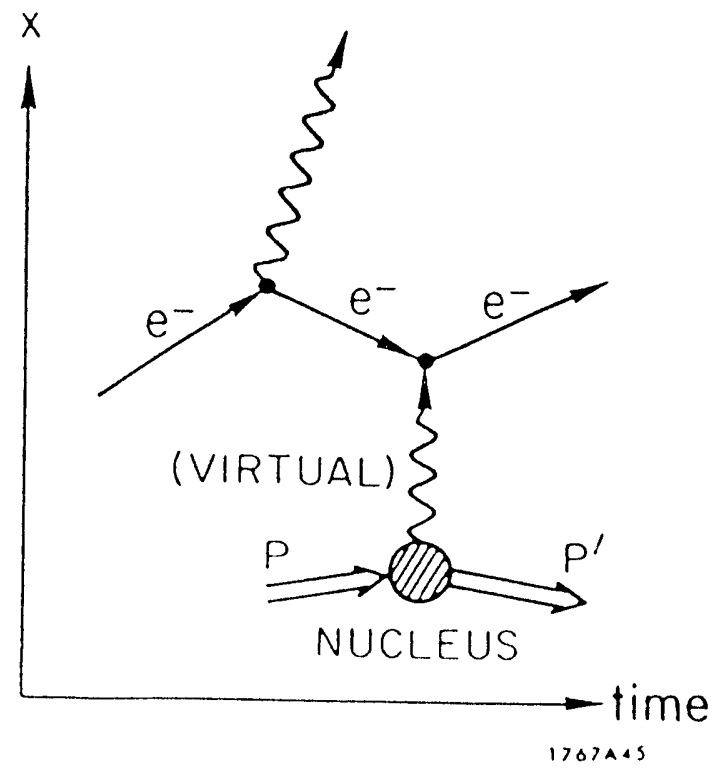

Fig. 7. Feyman diagrams for pair production and bremsstrahlung. 
If you reverse the $e^{+}$arrowhead in the pair production diagram, and change it to an $\mathrm{e}^{-}$traveling forward in time, then the diagram is identical to the bremsstrahlung diagram. In other words, the two processes are alike.

\subsection{Charged Particle Interactions}

There are two major processes for charged particles: collision (excitation and ionization) and radiative (bremsstrahlung). Collision losses can further be broken down into collisions close to the nucleus (hard collisions) and collisions at a distance (soft collisions). In general, collision processes dominate at low energies and radiative losses dominate at high energies. Figure 8 shows a plot of the fractional collision and radiative losses for water, air and lead ${ }^{[1]}$.

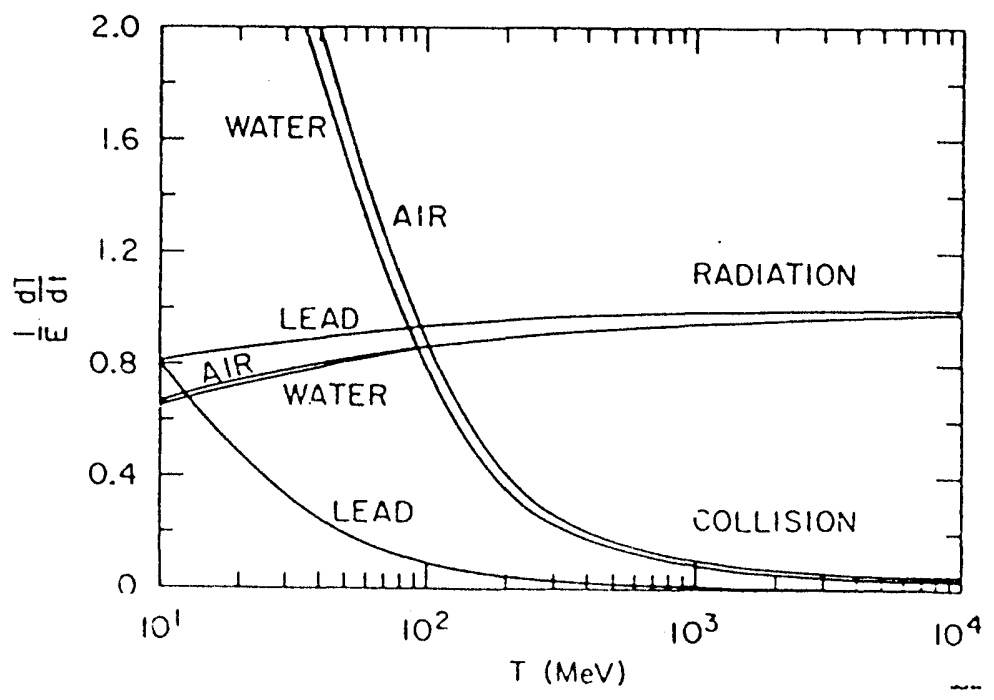

Fig. 8. Fractional energy loss per radiation length for electrons in water, air and lead ${ }^{\text {(11) }}$. 
The crossover point where radiative and collision losses are equal is called the critical energy and can be approximated by ${ }^{[12]}$

$$
\epsilon \simeq \frac{800}{Z+1.2}(\mathrm{MeV})
$$

The critical energy is about $10 \mathrm{MeV}$ for lead and $100 \mathrm{MeV}$ for water, which points to the energy at which EM showers become significant in those materials. The ratio between radiative and collision losses (i.e., stopping powers) can be approximated by $^{[10]}$

$$
\frac{\left.\frac{d T}{d x}\right|_{\text {rad }}}{\left.\frac{d T}{d x}\right|_{\text {col }}} \simeq Z\left(\frac{T}{1600 m_{0} c^{2}}\right)
$$

which shows that radiative losses increase linearly both with $Z$ and the electron kinetic energy. Therefore, for a beam of $33 \mathrm{GeV}$ electrons, bremsstrahlung is the most important interaction. It should be noted, however, that as the energies of the secondary electrons fall below the critical encrgy (11 MeV for tungsten), collision losses will begin to dominate.

Continuing with the bremsstrahlung process, whenever a charged particle traveling through matter is deflected from its path, or has its velocity changed, it may emit electromagnetic radiation whose energy is proportional to the deceleration received by the charged particle. This deceleration is caused by the influence of the electric field of the target nucleus on the particle. The radiation emitted is called bremsstrahlung (breaking radiation) and the high-energy (i.e., complete screening) cross section is expressed as ${ }^{[3]}$

$$
\begin{gathered}
\Phi_{\text {rad }}(T, k) d k=4 \alpha \frac{N_{0}}{A} Z^{2} r_{0}^{2} \frac{d k}{k}\left\{\left[1+\left(\frac{E^{\prime}}{E}\right)^{2}-\frac{2}{3} \frac{E^{\prime}}{E}\right]\left[\ln 183 Z^{-\frac{1}{3}}\right]\right. \\
\left.+\frac{1}{9} \frac{E^{\prime}}{E}\right\}\left(\mathrm{cm}^{2} \mathrm{~g}^{-1}\right),
\end{gathered}
$$


where

$$
\begin{aligned}
E & =\text { incident electron energy }(\mathrm{GeV}), \\
E^{\prime} & =\text { scattered electron energy }(\mathrm{GeV}), \\
k & =\text { photon energy }=E-E^{\prime}(\mathrm{GeV}) \\
Z & =\text { atomic number } \\
A & =\text { atomic mass }\left(\mathrm{g} \mathrm{\textrm {mol } ^ { - 1 }}\right) \\
\alpha & =\text { fine structure constant }=1 / 137 \\
N_{0} & =\text { Avogadro's number }=6.02 \times 10^{23}\left(\mathrm{~mol}^{-1}\right), \\
r_{0} & =\text { classical electron radius }=2.82 \times 10^{-13}(\mathrm{~cm}) .
\end{aligned}
$$

Two important observations can be made from this equation:

- The cross section is proportional to $Z^{2}$ - i.e., high- $Z$ materials are more likely to radiaie than low- $Z$ materials.

- The spectrum has a $1 / k$ shape-i.e., low-energy photons are more probable than high-energy ones.

At relativistic energies the scattered electron and the emitted photon tend to proceed in the same direction as the incident electron (i.e., the distribution is highly forward-peaked). As in the case of pair production described above, the differential cross section for bremsstrahlung is scaled in terms of a characteristic angle given by ${ }^{[10]}$

$$
\theta_{b r e m}=\frac{m_{0} c^{2}}{E},
$$

where $E$ is the (total) energy of the incident electron. Note again that $\theta_{b r e m} \leq 0.3^{\circ}$ for $E \geq 100 \mathrm{MeV}$.

Although the longitudinal development of the shower is dominated primarily by bremsstrahlung and pair production interactions, the lateral spread of the cascade is governed by elastic Coulomb scattering of the charged-particle component. The individual deflections are adequately described by the Mott formula ${ }^{[1.3)}$ which, as 
in the case of Rutherford scattering, has a $\theta^{-4}$ dependence. What this implies is that most of the scattering interactions lead to very small deflections. Small net deflections are generally the result of a large number of very small deflections; whereas, large net deflections are the result of a single large-angle scatter plus a number of very small deflections. The overall picture of charged-particle scattering in a medium can be viewed as a Gaussian-like distribution controlled by smallangle scattering, referred to as multiple scattering, superimposed onto which is a single-scattering (large-angle) tail ${ }^{\star}$.

Multiple scattering is best described analytically by the Fermi-Eyges model (e.g., see Chapter 3 of Kase and Nelson ${ }^{[1]}$ ), the form of which is a Gaussian measured in terms of a root mean square projected angle given by

$$
\theta_{r m s} \simeq \frac{15 \sqrt{t}}{E} \text { (radians) }
$$

where $t$ is the material thickness in radiation lengths and $E$ is the charged-particle energy in $\mathrm{MeV}$. If we now equate the characteristic angle of bremsstrahlung $\left(\theta_{b r e m}\right)$ to the rms scattering angle $\left(\theta_{r m s}\right)$ and solve for the thickness, we get

$$
t=\left[\frac{m_{0} c^{2}}{15}\right]^{2} \approx 0.001 \text { r.l. }
$$

In other words, charged particles scatter significantly prior to losing much energy by radiative interactions. In effect, this is a clear demonstration of the statement made earlier regarding the lateral spread of EM cascades being controlled by chargedparticle scattering.

* The region joining the two distributions in a smooth manner is often referred to as the plural scattering region ${ }^{(11)}$ 


\section{The EGS4 System}

\subsection{GenERAL}

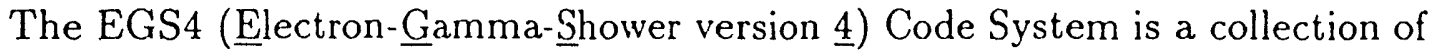
programs for the Monte Carlo simulation of coupled electron-photon transport ${ }^{[4]}$. It is designed to be used in any geometry and for energies from a few keV up to several $\mathrm{TeV}$. The system was first introduced ${ }^{[14]}$ in 1978 and was simply referred to as EGS3. Because of its versatility and relative ease-of-use, and because it has been checked against many experiments, EGS4 has been used rather extensively by the particle physics community in the development of shower counters and related detectors. There has also been a growing use of EGS4 by the medical physics community for modeling the transport of electrons and photons down to a few $\mathrm{keV}$ in complex geometries, including the human body.

\subsection{Capabilities and Features of the Code}

The following summarizes the main features of the EGS4 Code System:

- EGS4 is a package of subroutines plus block data with a flexible user interface.

- Electrons $( \pm)$ and photons can be transported in any element, compound, or mixture.

- The dynamic range of electron ( \pm ) kinetic energies goes from a few tens of keV up to a few thousand GeV.

- The dynamic range of photon energies lies between $1 \mathrm{keV}$ and several thousand $\mathrm{GeV}$.

- A data preparation code (PEGS4) creates data from cross-section tables for elements 1 through 100. The output is in a form for subsequent direct use by EGS4.

- The geometry for any given problem is specified by a user-uritten subroutine called HOWFAR. 
- The user scores and outputs information in the user-written subroutine called AUSGAB

- The EGS4 Code System is written in an extended FORTRAN language known as Mortran $3^{[15]}$.

- Variance reduction techniques are not built into EGS4 per se, but can be implemented by means of a macro facility available through Mortran3.

- Transport can be initiated from sources having spatial, angular, or energy distributions.

\subsection{Physics Processes in the EGS4 Code System}

The following physics processes are taken into account by the system:

- Electron $( \pm)$ bremsstrahlung.

- Positron annihilation at rest and in flight (annihilation quanta followed to completion).

- Multiple Coulomb scattering of electrons $( \pm)$ from nuclei (Molière model).

- Delta-ray production by means of $e^{-} e^{-}$(Møller) and $e^{+} e^{-}$(Bhabha) scattering.

- Continuous energy loss applied to electron ( \pm ) tracks between discrete interaction sites using a restricted stopping power (i.є., Bethe-Bloch formula including Sternheimer density effect correction).

- Pair production.

- Compton : cattering.

- Coherent (Rayleigh) scattering.

- Photoelectric effect (including K-edge fluorescent photon production). 


\subsection{Structure and Operation}

The overall interaction between user-written programs, the EGS4 code, and data is shown in Fig. 9.

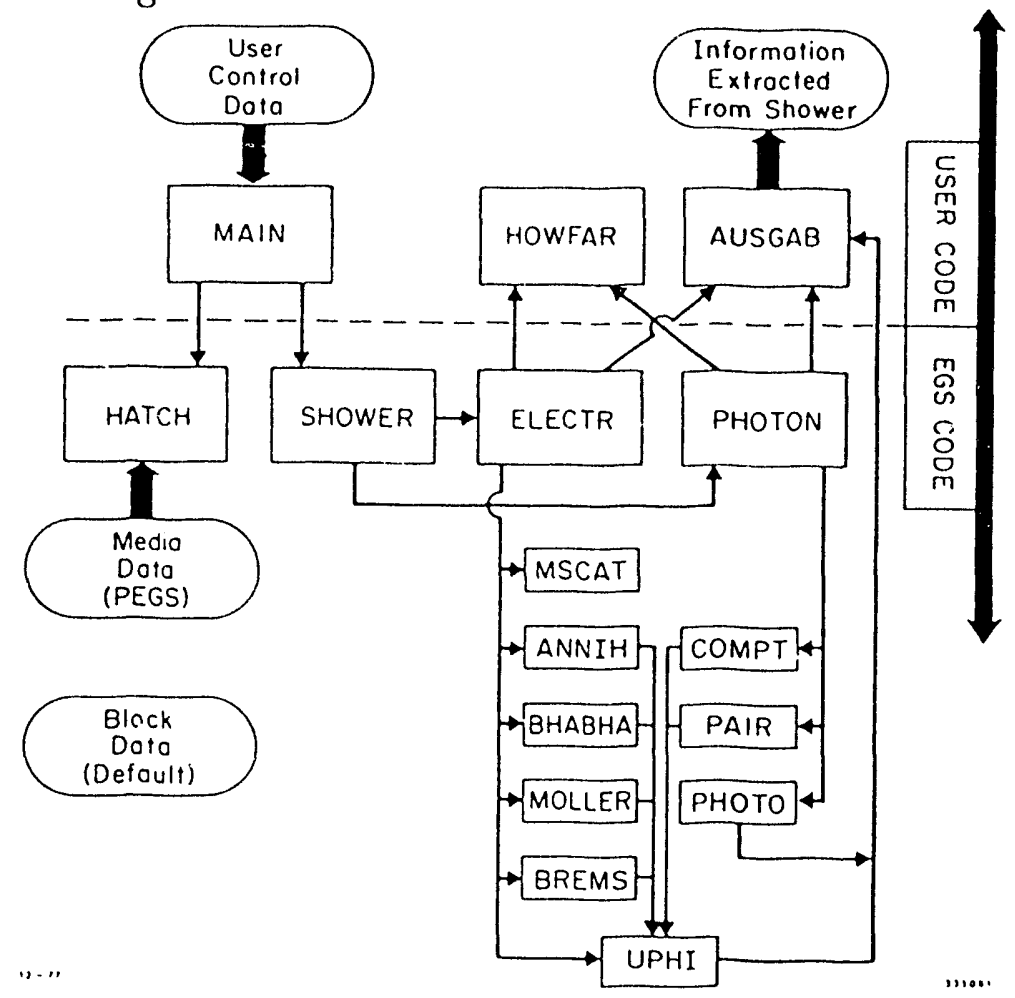

Fig. 9. EGS4 flow diagram ${ }^{[4]}$.

The dashed line represents the division between user-written subroutines (referred to as User Codes) and the default EGS4 subroutines. The MAIN program includes geometry initializations, materials used, energy cutoffs, and incident particle properties. MAIN then calls subroutine HATCH which "hatches EGS" by doing a once-only initialization and loading the data sets prepared by PEGS (Preprocessor for EGS). PEGS is a stand-alone preprocessor which constructs a linear fit over a large number of energy iniervals of the cross-section and branching-ratio data. The output of PEGS can be read directly by lis4. After the initialization, MAIN then calls SHOWER for each ECSSt history (i.e., the complete transport of one incident electron and all its progeny). 
In the course of tracking shower particles, subroutine AUSGAB may be called for a variety of reasons. AUSGAB is a subroutine that provides the user with the opportunity to score various quantities of interest (e.g., flux, dose, energy deposition, etc.). Subroutines ELECTR and PHOTON call HOWFAR which specifies the geometry of the problem.

A recent enhancement to the standard geometry that is available in EGS4 is the incorporation of the MORSE- $\mathrm{CG}^{[16]}$ combinatorial geometry package into HOWFAR ${ }^{[17]}$. This makes programming the geometry much easier but can increase the simulation time by factors of two or more. This package was not used in this analysis, however, due to the relatively simple geometry model assumed here.

The default version of EGS4 performs a straightforward analog Monte Carlo simulation. That is, each and every particle is followed to its completion (i.e., escape, energy cutoff, etc.). The particle weight, which is set to unity in the ana$\log$ mode, has been included as an argument in EGS to allow the user to take advantage of variance-reduction techniques such as importance sampling, splitting, Russian roulette, leading-particle biasing, etc. Extreme care must used when variance-reduction techniques are employed in EGS4, as in all Monte Carlo codes.

The EGS4 system is written in a programming language called Mortran $3^{[15]}$, which is then preprocessed into ANSI-standiard FORTRAN. The Mortran3 preprocessor itself is written in ANSI-standard FORTRAN. Therefore, this system can be loaded on any computer (including personal computers) which has an ANSIstandard FORTRAN compiler. The advantages of using Mortran3 over FORTRAN for producing code are summarized below:

- Free-field (column and card boundaries may be ignored).

- Alphanumeric labels of arbitrary length.

- Comments inserted anywhere in text.

- Nested block structure.

- Conditional statements which may be nested (IF, IF-ELSE, and ELSEIF). 
- Loops (repetitively executed blocks of statements) which test for termination at the beginning or end or both or neither (WHILE, UN'TIL, FOR-BY-TO, LOOP, and DO).

- EXIT (jump out of) any loop. Multiple assignment statements. Conditional (multiple) compilation. Program listing features include:

- Automatic printing of the nesting level.

- Automatic indentation (optional) according to nesting level.

- Abbreviations for simple I/O statements.

- Interspersion of FORTRAN text with Mortran text.

In addition, however, Mortran3 has an extensive macro-facility such that many FORTRAN steps can be accomplished in just a few Mortran steps. Users can also write their own macro's that redefine the default language. This makes the language "open- ended"-i.e., the user can make extensions to the language at any time to suit the problem.

The Mortran3 language is an example of why EGS4 is rclatively easy to use; the coding is easier to read than FORTRAN. This greatly simplifies writing new code and especially in modifying previously' written code.

\subsection{Graphics Capabilities}

EGS4 has been coupled with the SIAAC Unified Graphics System ${ }^{[18]}$ for displaying particle tracks on UGS77-supported devices ${ }^{[19]}$. It has also been modified to display the distribution of induced radioactivity in beam targets ${ }^{[20]}$.

This is accomplished by inserting the SHOWGRAF package onto the User Code and then making the CALL statements in the appropriate places in AUSGAB. The graphical output may be displayed directly onto an IBM 5080 color terminal which supports three-dimensional rotations, translations, and zooming. SHOWGRAF 
can also output graphics into files for printing on a $\mathrm{PC}$ display using a postprocessor system called EGS4PL ${ }^{[21]}$. Figure 10 is an example of an output from SHOWGRAF.

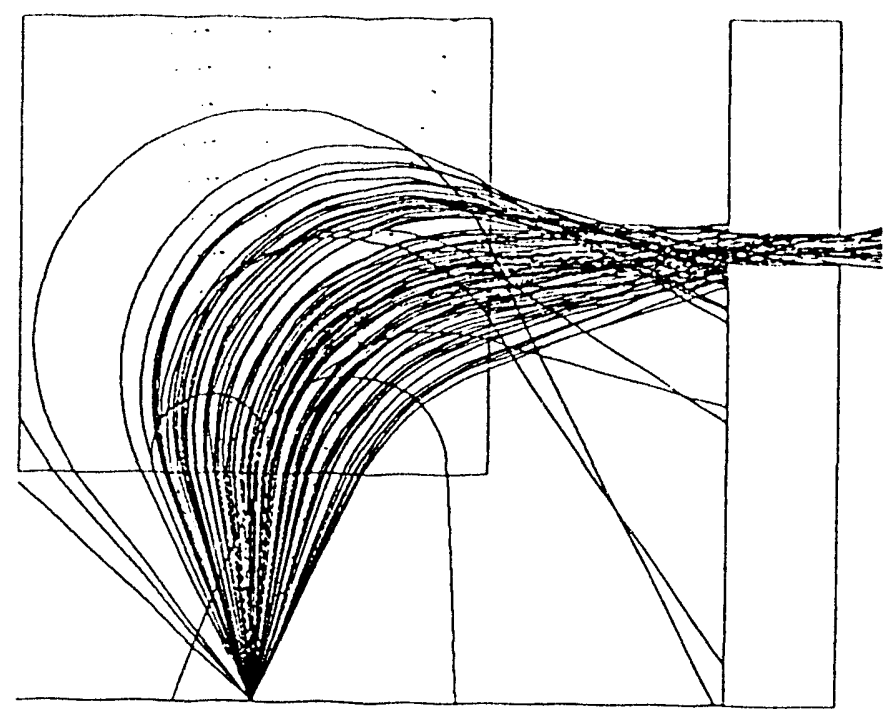

Fig. 10. EGS4 simulation of $8.5 \mathrm{MeV}$ electrons scattering in a $0.38-\mathrm{mm}$ copper foil (100 events). A $2.6 \mathrm{kG}$ magnetic field focuses the electrons through a lead slit. Solid lines are electrons and dots are photons.

\subsection{BENCHMARKS}

As stated earlier, EGS4 is used extensively throughout the world in both the high-energy physics and the medical physics communities. Perlaps the most impressive benchmarks are performed by the users and the results of these benchmarks can be demonstrated by the growing number of EGS4 users. A recent book entitled Monte Carlo Transport of Electrons and Photons ${ }^{[22]}$ provides a good collection of these benchmatks that have been performed by various authors. 
Figure 11 shows an example of the excellent agreement between a physical experiment and the corresponding EGS4 simulation ${ }^{[23]}$.

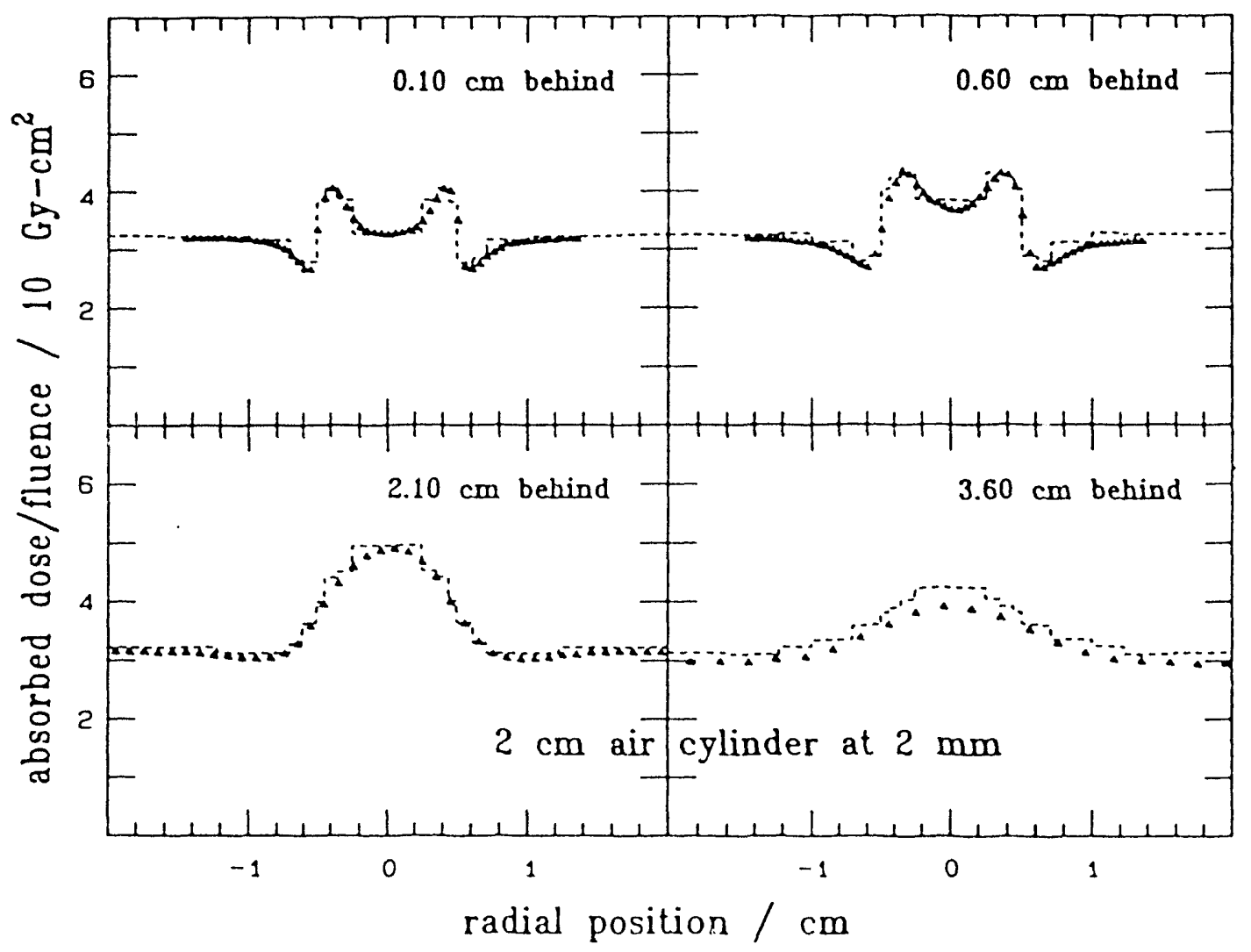

Fig. 11. Radial Dose Profiles Behind an Air Cylinder in a Water Phantom irradiated by a 20-MeV beam of Electrons ${ }^{[23]}$.

Another important benchmark, demonstrating the close agreement between EGS and experiment, is the recent thesis by Faddegon ${ }^{[24]}$. 


\section{ETRANS}

ETRANS ${ }^{*}$ is a Pascal/FORTRAN/Mortran program that follows electrons $( \pm)$, one at a time, through an electromagnetic field having rotational symmetry about the incident electron beam direction. The program was written at $\mathrm{SLAC}^{[25]}$ to model the transport of positrons in the capture section (i.e., $\mathrm{e}^{+}$collector) downstream of the SLC positron target. The capture section consists of the following:

1. A set of magnetic solenoids.

2. A flux concentrator which provides a very high, rapidly varying field.

3. Accelerator sections.

The experimental results agree quite well with predictions by ETRANS, using EGS-generated $\mathrm{e}^{+}$input, for the current SLC target scheme.

\section{SLC Positron Target}

The SLAC Linear Collider (SLC) is an electron-positron machine system designed to accelerate and collide two $50 \mathrm{GeV}$ beams (see Fig. 12). Electrons are emitted from a thermionic gun and accelerated to $1 \mathrm{GeV}$. A splitter magnet deflects electrons and positrons into separate damping rings where synchrotron radiation reduces their transverse emittance. One positron bunch ${ }^{\dagger}$ and two electron bunches are ejected and accelerated down the linac. The positron bunch and the first of the electron bunches are accelerated to about $50 \mathrm{GeV}$. The two bunches are separated and transported around the arcs, bringing them into collision at the interaction point. The bunches are then deflected into beam dumps. The second electron bunch is extracted at the $2 / 3$ point in the linac, where its energy is $33 \mathrm{GeV}$, in order to produce positrons. Positrons are then captured, accelerated, and returned to the front end of the linac for further acceleration into the damping ring.

* Not to be confused with the FTRAN Monte (arlo program ${ }^{123}$.

$\dagger$ A bunch is typically 1 to $2 \times 10^{10} \mathrm{e}^{+}$or $\mathrm{e}^{-}$ 


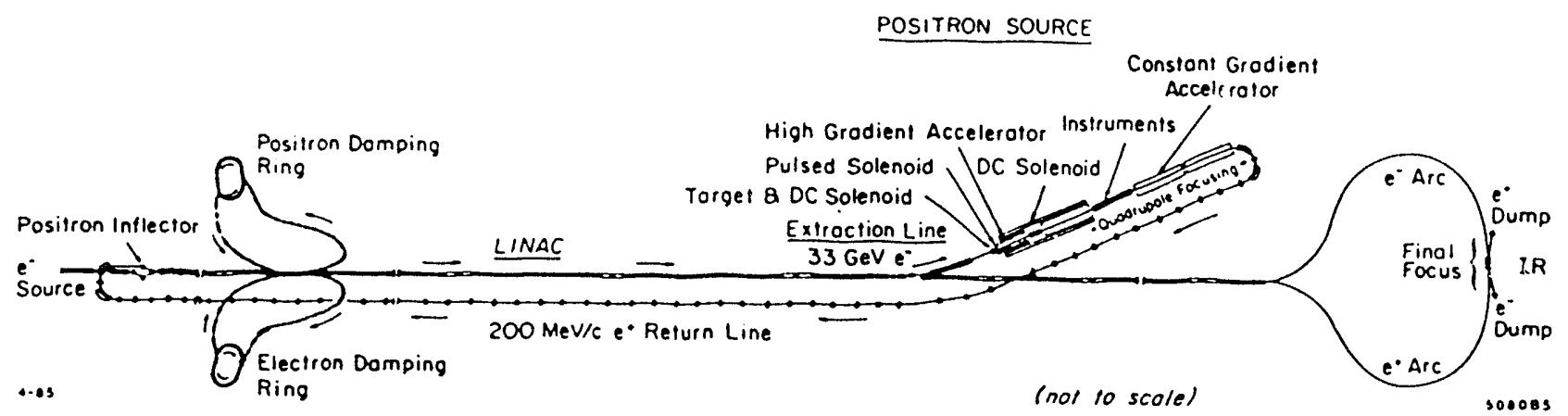

Fig. 12. Schematic of the Stanford Linear Collider (SLC) (taken from Bulos ${ }^{|26|}$ ).

Figure 13 shows a schematic of the current SLC positron source and subsequent magnetic capture system ${ }^{[27]}$.

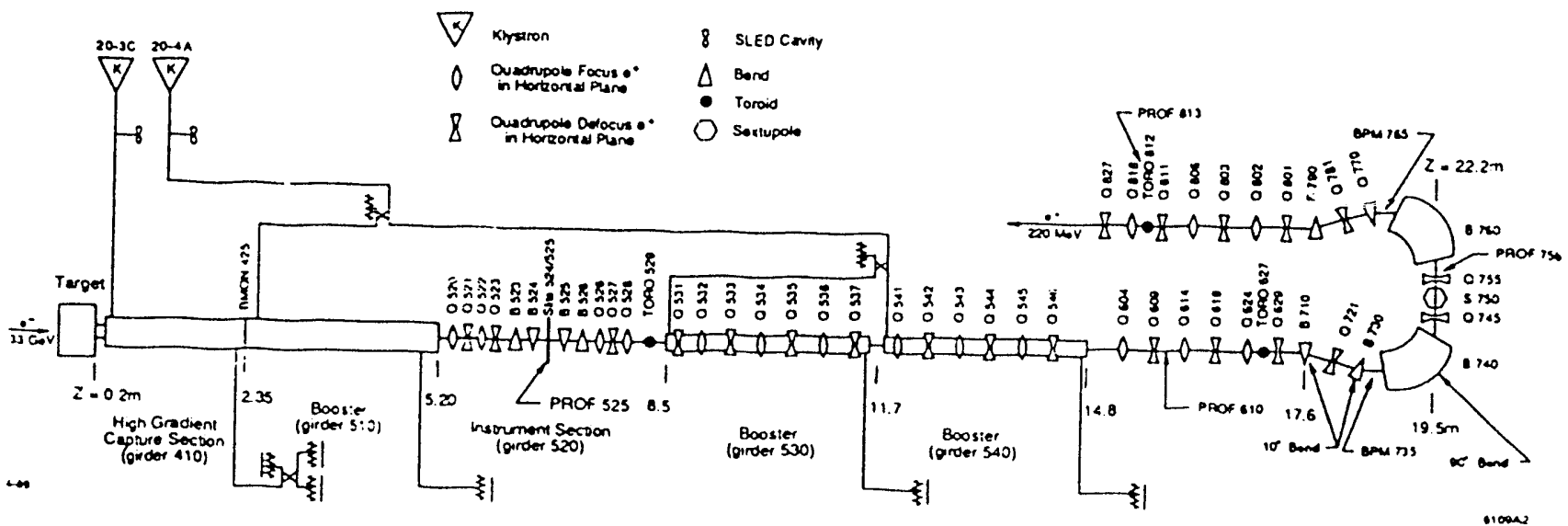

Fig. 13. Schematic of positron source and capture section ${ }^{[27]}$.

A $33 \mathrm{GeV} \mathrm{e}^{-}$beam is incident from the far left producing positrons via an EM cascade shower in a tungsten-alloy target. Positrons which are produced within an acceptable phase space $(\bar{x}, \bar{p}, \mathrm{t})$ are captured, accelerated, transported back to the front end of the two-mile accelerator, accelerated to $50 \mathrm{GeV}$, and then transported to the SLC interaction region. 
A closeup view of the SLC target ${ }^{[26][27][28-30]}$ and flux concentrator are shown in Fig. 14. The current target is about a centimeter in diameter and is 6-r.l. long $(2.1-\mathrm{cm}$.). This length corresponds to roughly shower maximum $(\approx 7$-r.l.) and produces the maximum number of positrons. This target can be roughly modeled as a semi-infinite slab when comparing the transverse dimension of the target to the lateral spread of the cascade shower.

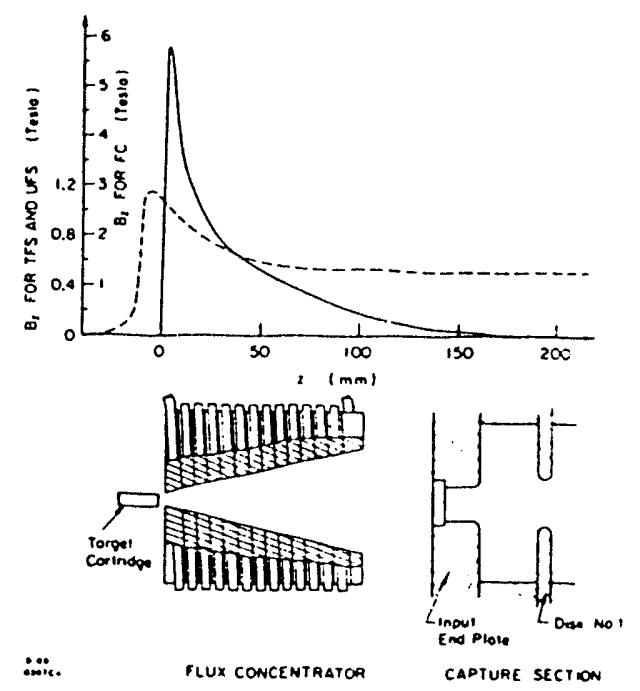

Fig. 14. Front portion of capture section ${ }^{[27]}$.

The target is closely followed $(\approx 3-\mathrm{mm})$ by a strongly-pulsed magnetic field $(30 \mathrm{kA}, 120 \mathrm{~Hz})$, referred to as the flux concentrator. It's field strength is shown as the solid line in Fig. 14. The target and flux concentrator are surrounded by a DC-field solenoid (not shown in the figure), whose field strength, as a function of axial distance, is shown as the dashed line.

The positrons emerge from the target with a small longitudinal spread and a large angular dispersion, but the capture section itself has a relatively small angular acceptance. The purpose of this focusing system is to collect positrons by performing a phase transformation of transverse momentum to longitudinal momentum. It is designed to transform a 2 -mm $\times 2.5 \mathrm{MeV} / \mathrm{c}$ transverse momentum beam into an 8 -mm $\times 0.6 \mathrm{MeV} / \mathrm{c}$ beam. 
Figure 15 shows the energy spectrum of positrons produced in the target, accepted by the capture system, and accepted into the damping ring for subsequent acceleration to $50 \mathrm{GeV}^{[26]}$. As shown, the current target has a yield of 2.5 positrons for every incident electron.

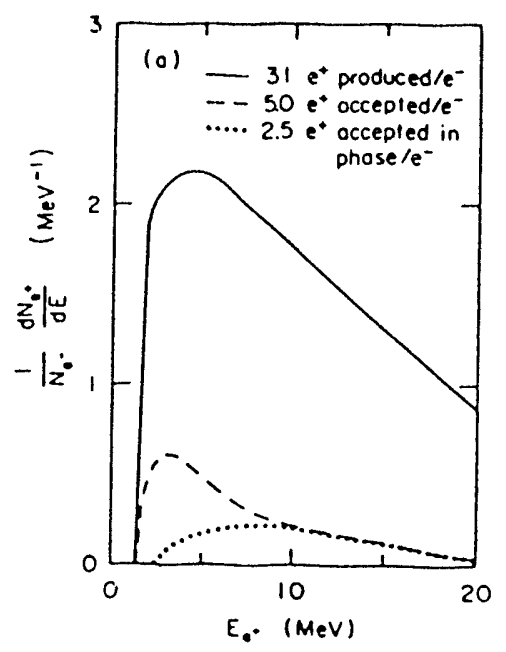

Fig. 15. Positron energy spectrum ${ }^{|26|}$.

\section{Proposed Positron Target}

\subsection{General}

It is estimated that the majority of the positrons captured by the present SLC target are produced close to the end of the target; that is, most of the positrons produced within the target are reabsorbed before escaping. From Fig. 15 there are about $31 \mathrm{e}^{+}$produced off the end of the target per incident $\mathrm{e}^{-}$, yet only about $5 \mathrm{e}^{+} / \mathrm{e}^{-}$are accepted by the capture section.

As we ha.e seen earlier (see Eqn. 2.1), the total number of particles created $\left(\mathrm{e}^{+}, \mathrm{e}^{-}, \gamma\right)$ in the cascade is approximately given by $E_{0} / E_{c u t}$. EGS4 calculations, however, predict a maximum $e^{+} / e^{-}$production inside the target of only a few hundred, indicating that Eqn 2.1 overestimates significantly for low-cutoff energies. 
In spite of this, there is considerable incentive to investigate different target designs in order to enhance the $e^{+} / e^{-}$yield by collecting some of the positrons from within the target, as well as those escaping the end. Although there may be geometries which are even better, this paper investigates the possibility of small diameter wires as a first approach to increasing the yield.

\subsection{EGS4 User Code}

An EGS4 User Code was written to simulate the production of positrons in thin tungsten wires. The following enhancements were made to the system:

- Implemention of the PRESTA ${ }^{[31]}$ algorithm which optimizes the allowable electron step-size. This insures that the step-size is just small enough to avoid artifacts and still large enough that computing time is not wasted.

- Implemention of an algorithm for angular sampling of the bremsstrahlung photons $^{[32]}$. In the default version of EGS4, bremsstrahlung photons are given a fixed production angle corresponding to $\theta=\frac{m c^{2}}{E}$, where $E$ is the incident electron energy and $m c^{2}$ is the electron rest mass energy. This assumption is reasonable for high-energy beams incident on thick targets since, as we have seen earlier, multiple scattering completely dominates. However, this assumption has been shown to break down for thin targets and for electrons with $E_{0} \leq 10 \mathrm{MeV}^{[32]}$. The new algorithm is based on the formula for the bremsstrahlung photon angular distribution developed by Koch and Motz ${ }^{[33]}$.

- Implemention of SHOWGRAF ${ }^{[21]}$ for displaying particle tracks on SLAC Unified Graphics ${ }^{[18]}$ supported devices.

- Modification of the User Code to "tag" the birthplace of all positrons crealed inside the target. This unique location is carried throughout the shower process and is available for outputing by the user in subroutine AUSGAB. 


\subsection{Wire Target Yields}

Figure 16 shows the relative positron yield as a function of wire radius for a fixed (6-r.l. high-Z) target ${ }^{[34]}$. The yields are normaiized to the calculated yield from a 6-r.l. semi-infinite slab of tungsten, which is in good agreement with the present SLC positron target.

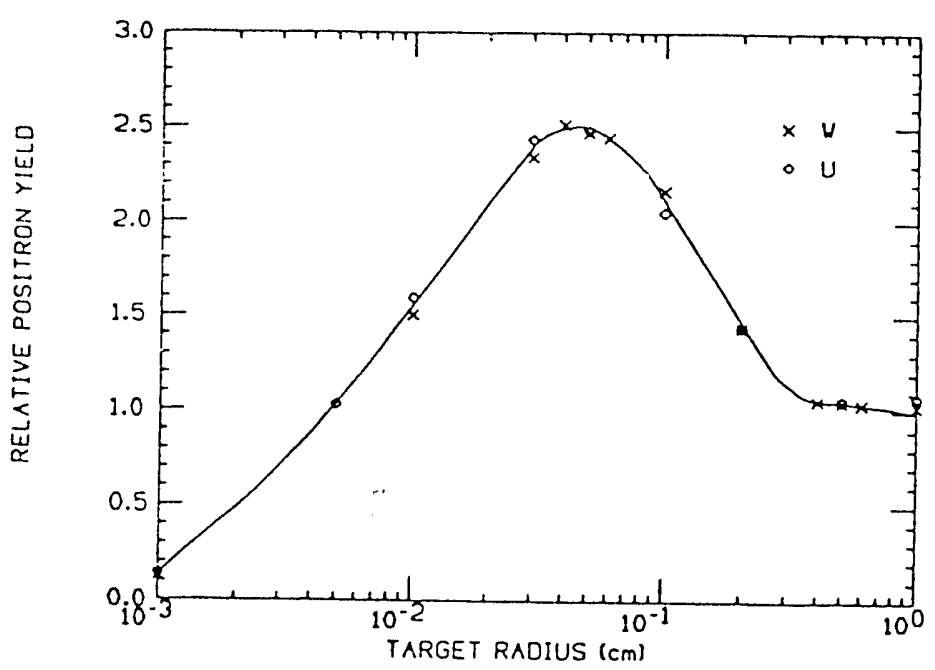

Fig. 16. Relative yield from a 6-r.l. tungsten wire vs radius.

The yield in Fig. 16 is defined as any positron which passes through a plane at the downstream end of the target regardless of its distance from the center of the wire, its transverse momentum, or its arrival time at the plane. This figure can be explained as follows. At small radii, photons and charged particles escape too early from the sides of the target, precluding a substantial electromagnetic shower development. At large radii the shower reaches maximum development but many of the particles are absorbed in the target itself. Note that after about $0.4-\mathrm{cm}$ radius, the yield flattens out and the target can be considered as semi-infinite. The yield reaches a maximum at $1-\mathrm{mm}$ where the yield is 2.5 times greater than for the current (semi-infinite) target use in the SIC:

After fixing the target diameter at $1-m m$, the target length was then varied to see if there would be any further increase in yield. Figure 17 shows the relative yield 
from 1-mm tungsten targets of varying lengths and with several different types of cladding materials. As this figure shows, a 10-r.l. wire suspended in vacuum has a yield which is a factor of 4.3 times that of the current SLC target.

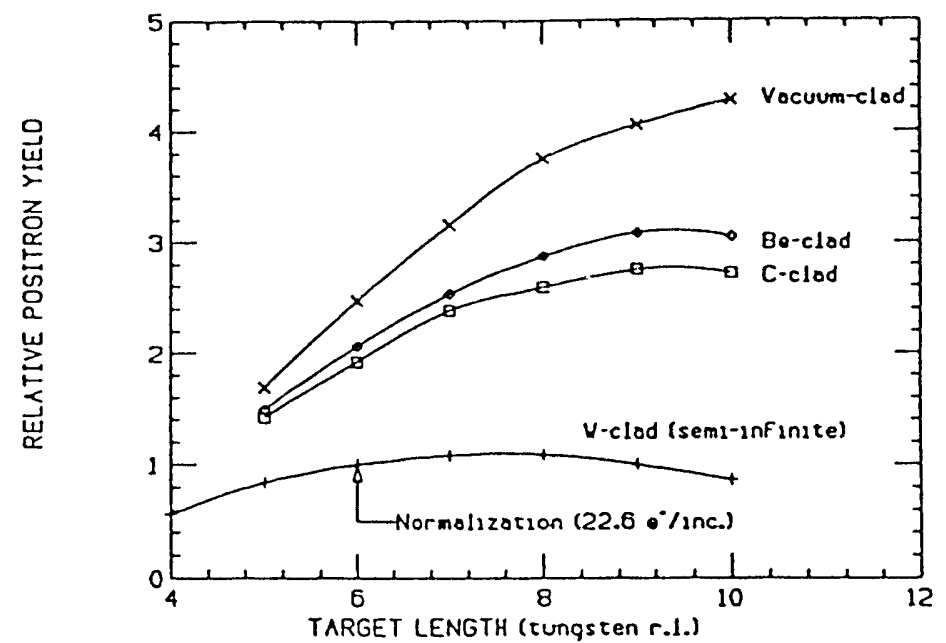

Fig. 17. Length $v s$ yield from 1-mm diameter tungsten wires (with various cladding).

However, since the majority of these positrons are leaking from the sides, they may not be within an acceptable phase space for the capture section downstream. ETRANS was used to model the transport of positrons through the capture system, using the output of EGS4 as input. ETRANS calculated a relative yield of about 2.5 ( $v s 4.3$ ), meaning that about $40 \%$ of the increased yield coming from the wire target is contained within a phase space that is unacceptable to the current capture system.

A new capture strategy ${ }^{[34]}$ suggests a way of increasing the yield of either target-wire or slab. This involves

1. back-phasing the positrons in a low-gradient field, instead of the present high-gradient flux concentrator, to minmize any further phase spread,and

2. the elimination of a half-meter instrumentation section to reduce the drift space. 
The results are summarized in Table 1 and in Fig. 18 which, in addition, shows where losses occur all along the capture system.

\section{Table 1}

Summary of Target Yields $\left(\mathrm{e}^{+} / \mathrm{e}^{-}, 2 \mathrm{MeV}\right.$ cutoff).

\begin{tabular}{|c|c|c|}
\hline & Slab Target & Wire Target \\
\hline Present Capture & 2.7 & 6.8 \\
New Capture & 4.3 & 9.3 \\
\hline
\end{tabular}

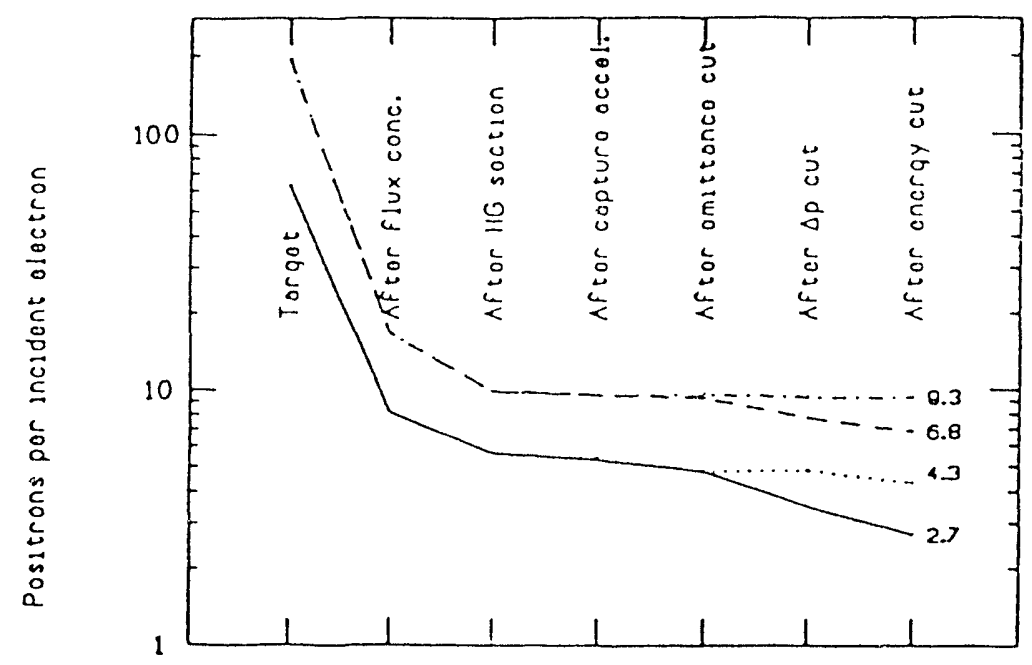

Fig. 18. Location of losses in the positron capture system.

To better understand where positrons are produced in the target the liser Code was modified using the TRANSFER PROPERTIES macro available in EGS. This macro contains information for each individual particle in the shower. The information is retained with the particle throughout the shower and is available for use at any point. The birthplace $\left(x_{t a g}, y_{t a g}, z_{t a g}\right)$ of all positrons produced in the target was added to this marro. Also adder? was a unique identifier for each positron, consisting of the incident electron event number and the positron nunber separateri by a decimal mine. For example, the sixth positron created 
by the second incident electron would have an identifier of 2.006. This identifier was read in with the ETRANS input file (from EGS4) and also output with the positrons accepted by ETRANS. The ETRANS output was then used as EGS4 input. Namely, the showers were initiated again with the identical random number seed used to create the rays initially. The identifier of every positron created in the shower was compared with the identifier of the accepted positrons. If they matched, the location, energy, momentum, etc. were output for analysis. If the ID's did not match, the particle was discarded. In this way, it is known during the shower which positrons will be accepted by the capture section. This is summarized in Fig. 13.

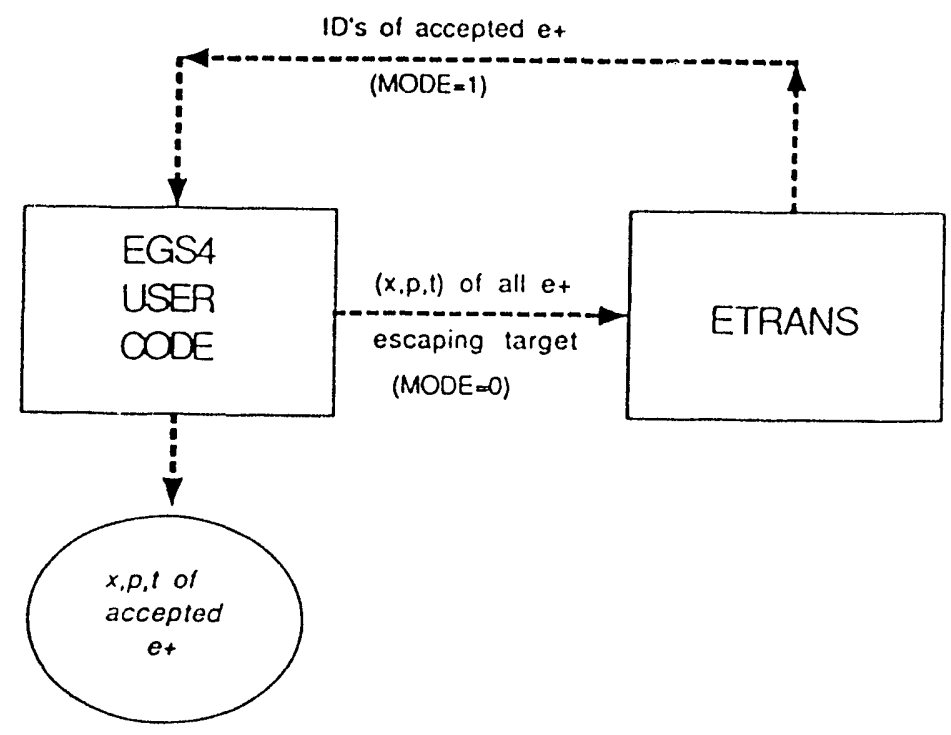

Fig. 19. EGS4 mode switch.

Figures 20 and 21 show the birthplace in the slab and wire targets, respectively, of positrons which have been accepted by ETRANS. Both figures are for 100 incident $33 \mathrm{GeV}$ electrons. Figure 20 clearly shows that most of the captured positrons are produced in the vicinity of the downstream face of the slab target. Those few positrons which are produced near the front face of the target are probably high-energy positrons while those at the downstream end probably have much lower energy. 


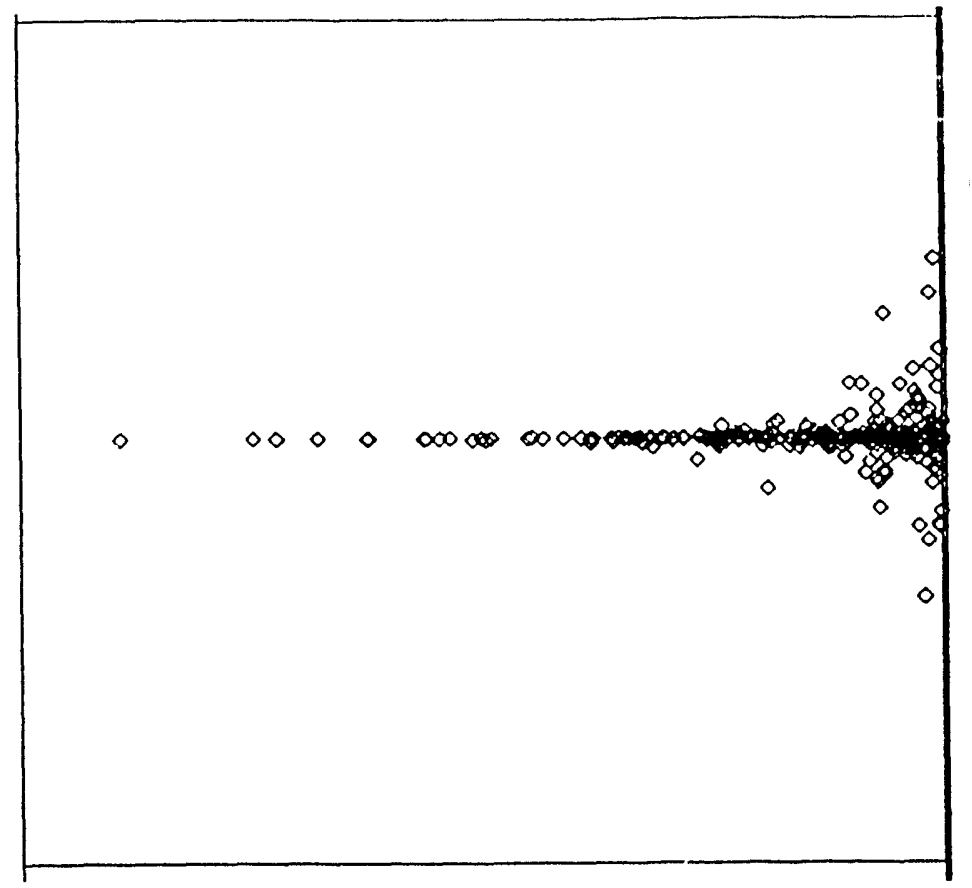

Fig. 20. Positron birthplaces in a slub target: 100 incident $\mathrm{e}^{-}, 352$ accepted $\mathrm{e}^{+}$.

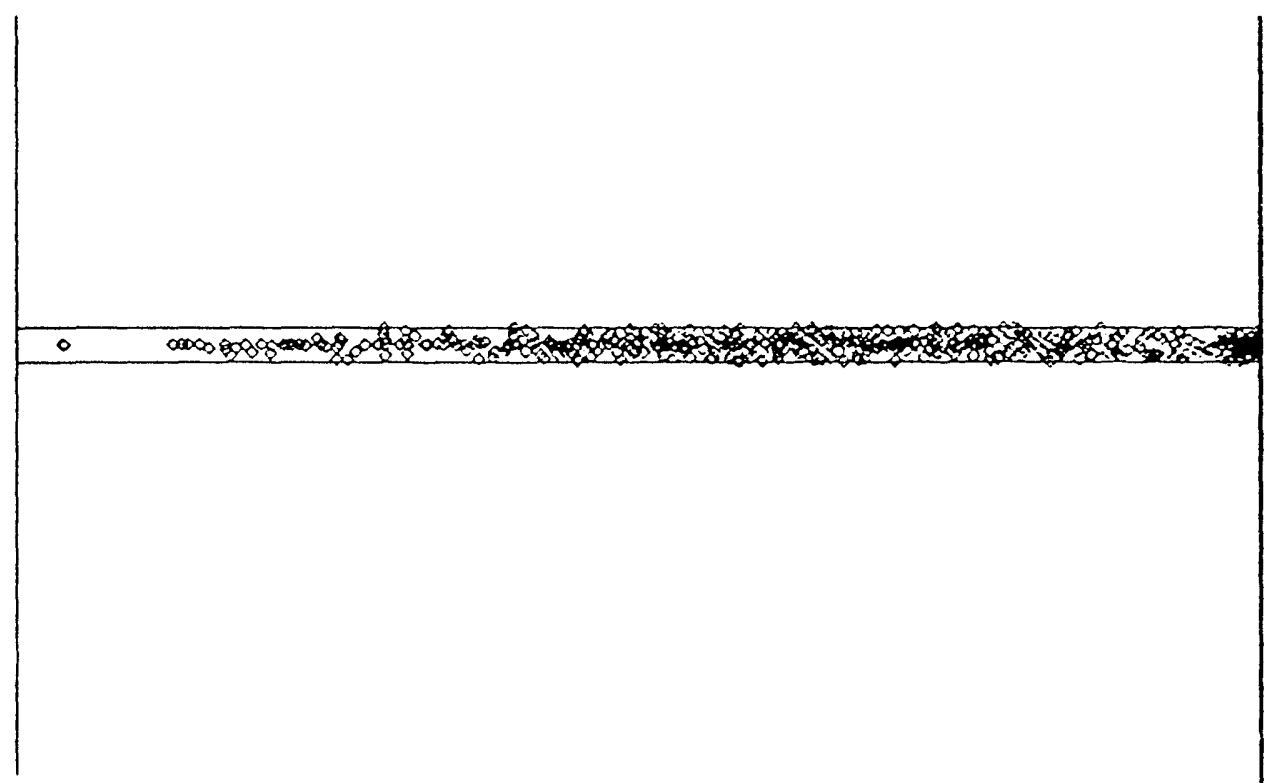

Fig. 21. Positron birthplaces in a wire target: 100 incident $\mathrm{e}^{-}, 793$ accepted $\mathrm{e}^{+}$.

On the other hand, the wire-target picture (Fig. 21) shows that accepted positrons are produced somewhat more uniformly along the wire. These positrons are escaping from the side of the target but their transverse momentum $p_{t}$, is still small enough that they are within the acceptance of the capturs section. 
Figure 22 shows more complete characteristics of the positrons in the target accepted by the capture section, for both slab and wire targets.
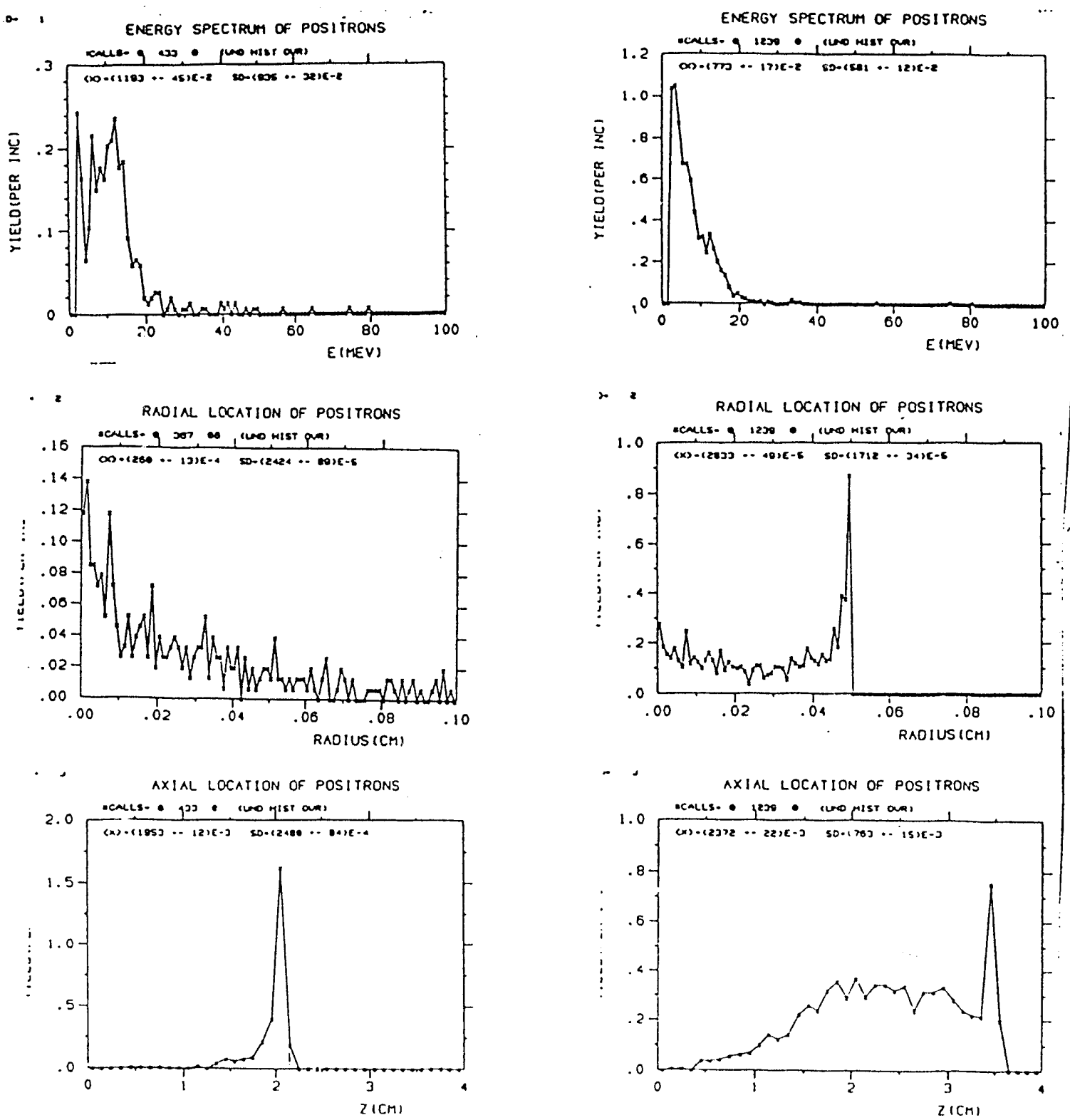

Fig. 22. Energy spectrum and radial-axial locations of accepted positrons inside targets. The slab target is shown in the left column (a-c) and wire target in the right column (d-f). 


\subsection{Alternative Geometries}

Figure 23 is a plot of the birthplace of 680 positrons inside a semi-infinite slab target that were accepted by ETRANS, which suggests that perhaps a smalldiameter, conc-shaped target may enhance the yield. This is reasonable from a cascade shower viewpoint since the lateral spread of the shower increase as it develops. This is clearly demonstrated in the SHOWGRAF plots given in Fig. 24 showing particle tracks created inside a 10-r.l. wire target.

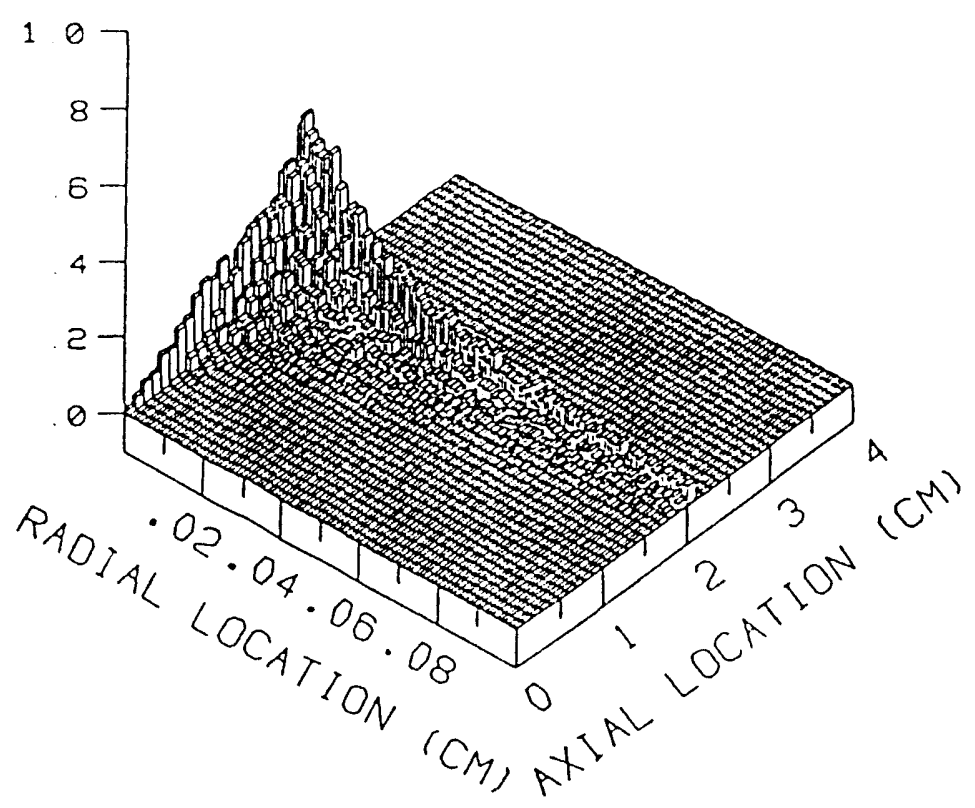

Fig. 23. Birthplace of positrons in 6-r.l. slab target which have been accepted by the capture system. 


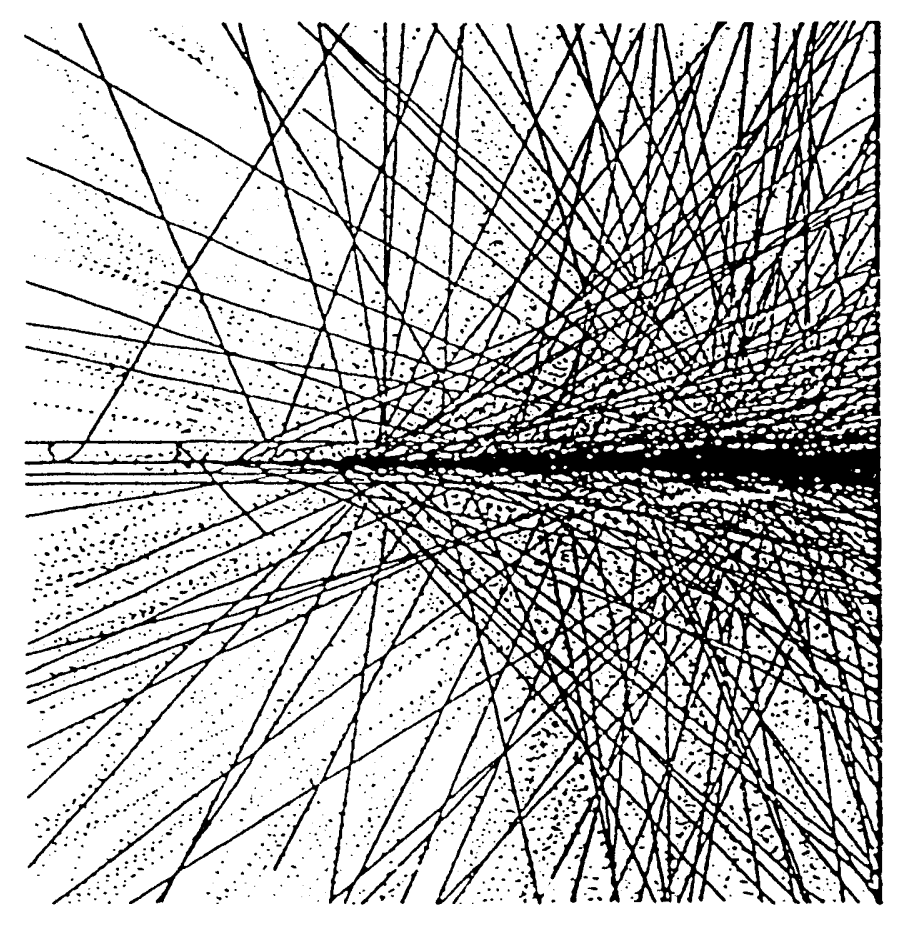

Fig. 24. EGS4/SHOWGRAF shower simulations (solid lines are charged particles and dotted lines are photons).

\section{Thermal Heating}

There are three important quantities which must be determined for the positron target: temperature rise per pulse, the maximum stress at the central core and the steady-state temperature,. The radial stresses are usually determined from the temperature rise per pulse, which is obtained from the fractional energy-loss per unit volume, $d E / E_{0} d V$, as follows ${ }^{[23-37]}$ :

Temperature rise per pulse, $\Delta T_{p}\left({ }^{\circ}(/ p u l s e)\right.$ :

$$
\Delta T_{p}=\left(\therefore E_{0} \frac{1}{\rho E_{0}} \frac{d V}{d V}\right.
$$


where

$$
\begin{aligned}
\rho & =\text { material density }\left(\mathrm{g} / \mathrm{cm}^{3}\right), \\
C_{p} & =\text { heat capacity } \approx \frac{6.0}{A}\left(\mathrm{cal} / \mathrm{g}^{\circ} \mathrm{C}\right), \\
N & =\text { electrons/pulse, } \\
E_{0} & =\text { incident beam energy }(\mathrm{MeV}), \\
A & =\text { atomic weight }(\mathrm{g} / \mathrm{mole}), \\
C & =1.6 \times 10^{-13} / 4.184(\mathrm{cal} / \mathrm{MeV}) .
\end{aligned}
$$

Maximum Radial Stress $(r=0), \sigma_{r}$ (psi):

$$
\sigma_{r}=\frac{a E_{y} \Delta T_{p}}{2\left(1-\nu_{p}\right)}
$$

where

$$
\begin{aligned}
\nu_{p} & =\text { Poisson ratio }(0.25 \text { to } 0.30), \\
\alpha & =\text { coefficient of thermal expansion }\left({ }^{\circ} \mathrm{C}^{-1}\right), \\
E_{y} & =\text { Young's modulus (psi). }
\end{aligned}
$$

\subsection{Temperature Rise/Pulse}

A previously written EGS4 User Code ${ }^{[3 *]}$ has been modified to produce the fractional energy deposition per unit volume. $\frac{1}{E_{0}} \frac{d E}{d V}(r, z)$, which we will refer to as the energy-deposition density. This User Code was written to determine the pulse temperature rise in radially-large slabs (e.g., beam dumps, collimators, targets, etc.). EGS4 produces the energy-deposition density for a $\delta$-function beam (i.e., pencil beam). The user can write a routine tr sample the beam over an appropriate distribution to obtain the proper beam shape but a large amount of execution time would be required to get enough statistics in the onter radial bins. What was done instead was to take the energy-deposition density and convolute it analytically with

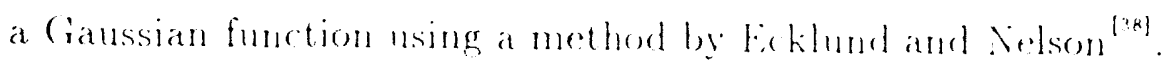


The general form for a 1-dimensional Gaussian distribution is

$$
f(x)=\frac{1}{\sqrt{2 \pi} \sigma} \int_{-\infty}^{\infty} e^{-\frac{(x-\bar{x})}{2 \sigma^{2}}} d \bar{x}
$$

If we assume that the energy-deposition density of the pencil beam is given by

$$
W_{0}(r) \equiv \frac{1}{E_{0}} \frac{d E}{d V}
$$

then the convoluted energy-deposition density is

$$
W_{\sigma}(r)=f * W_{0}=\int f(x) W_{0}(x) d x
$$

For a 2-dimensional Gaussian distribution in radial coordinates $(\bar{x}=\bar{r} \cos \bar{\theta}$, $\bar{y}=\bar{r} \sin \bar{\theta})$,

$$
\begin{aligned}
W_{\sigma}(r) & =\frac{1}{2 \pi \sigma^{2}} \int_{0}^{\infty} d \bar{r} \int_{0}^{2 \pi} d \bar{\theta} \bar{r} e^{-\left(\frac{\left.r^{2}+\bar{r}^{2}-2 r \bar{r} \cos (\theta-\bar{\theta})\right)}{2 \sigma^{2}}\right.} W_{0}(\bar{r}) \\
& =\frac{1}{2 \pi \sigma^{2}} \int_{0}^{\infty} \bar{r} d \bar{r} e^{\frac{-(r-\bar{r})^{2}}{2 \sigma^{2}}} W_{0}(\bar{r}) \int_{0}^{2 \pi} d \bar{\theta} e^{-\frac{r \bar{r}}{\sigma^{2}}(1-\cos \bar{\theta})}
\end{aligned}
$$

where we have taken $\theta=0$ without loss of generality.

Since the EGS4 output is in the form of a histogram averaged over radial bins, we have

$$
W_{\sigma}(i) \equiv \int_{r_{1}}^{r_{i+1}} W_{\sigma}(r) r d r
$$

The convoluted distribution with the same binning is then

$$
W_{\sigma}(i)=\sum_{j} M_{i j} W_{\sigma}(j)
$$


where

$$
M_{i j}=\frac{1}{\pi \sigma^{2}\left(r_{i+1}^{2}-r_{i}^{2}\right)} \int_{r_{1}}^{r_{i+1}} \int_{r_{j}}^{r_{++1}} r d r \bar{r} d \bar{r} e^{\frac{-(r-\bar{r})^{2}}{2 \sigma^{2}}} \int_{0}^{2 \pi} e^{-\frac{r \bar{r}}{\sigma^{2}}(1-\cos \bar{\theta})} d \bar{\theta} .
$$

The integral over $\bar{\theta}$ can be reduced to a modified Bessel function, $\mathrm{I}_{0}$. The double integration is done numerically, taking special care (along $i=j$ ) to provide the quadrature routine with an integrand that is not ill-behaved over the bin in question. The above equation assumes that the energy-deposition density does not vary significantly over the width of each bin.

Several programs were written ${ }^{[38]}$ to create, check and use the matrix elements. They are summarized below in 'Table 2 (and included here for any future reference).

Table 2. Summary of programs used for Gaussian convolution of pencil beam.

\begin{tabular}{|c|l|}
\hline Name (filename-filetype) & \multicolumn{1}{|c|}{ Purpose } \\
\hline UCDEDV MORTRAN & $\begin{array}{l}\text { EGS4 User Code to calculate energy-deposition } \\
\text { density for } W_{0}(r) .\end{array}$ \\
\hline DEDVM MORTRAN & $\begin{array}{l}\text { Program to create matrix elements for Gaussian } \\
\text { convolution. }\end{array}$ \\
\hline DEDVMC MORTRAN & $\begin{array}{l}\text { Program which checks } \sum_{j} M_{i j}=1.0 \text { for any } \\
\text { i-bin. }\end{array}$ \\
\hline DEDV DATA & $\begin{array}{l}\text { Data file containing matrix elements followed by } \\
\text { energy-deposition densities }(i . e ., n-s e t s \text { for n-runs } \\
\text { on the computer }) .\end{array}$ \\
\hline DEDV MORTRAN & $\begin{array}{l}\text { Program which reads in the above data file and } \\
\text { outputs the convoluted energy-deposition density } \\
\text { in the form of plots. }\end{array}$ \\
\hline
\end{tabular}


The temperature rise per pulse (and $\frac{d E}{E_{0} d V}$ ) for the convoluted beam are shown in Figs. 25-34 for 1-r.l. to 10-r.l. 'The maximums are summarized in Fig. 35. Figure 36 shows the longitudinal energy-deposition density (per r.l.) as a function of target length (r.l.) for a semi-infinite target $(r \rightarrow \infty)$.

The current SLC beam is about 0.6 to $0.8-\mathrm{mm}$ on the slab target. A rather striking trend can be noticed from these plots: for a beam size between 0.5 to $1-\mathrm{mm}$, the temperature rise per pulse is almost perfectly flat across the wire radius, but varies by a factor of 10-20 along the length of the wire. The slab target energydeposition density can vary by at least three orders of magnitude over a radius of a few centimeters, and perhaps a factor of 30-40 from front to back ${ }^{[38]}$. This demonstrates a possible advantage of the wire over the slab target. Eq. 7.2 shows that the radial stresses are directly proportional to the temperature rise per pulse, and therefore proportional to the energy-deposition density. This demonstrates why the radial stresses create such a problem for thick targets. While the axial stresses may be of the same order of magnitude for both targets, there will be a large difference in the radial stresses. As shown in Figs. 25-34, the wire target seems to be uniformly heated in the radial direction and may not suffer the effects of large radial stresses. Remember that $0.5<\sigma<1-\mathrm{mm}$ and the optimum wire target diameter is $1.0-\mathrm{mm}-$ i.e., the target and beam are about the same size. Therefore, the radial stresses for the wire target are considered small when compared to the slab target and are neglected. The important question then becomes: "What about the steady-state temperature?" 

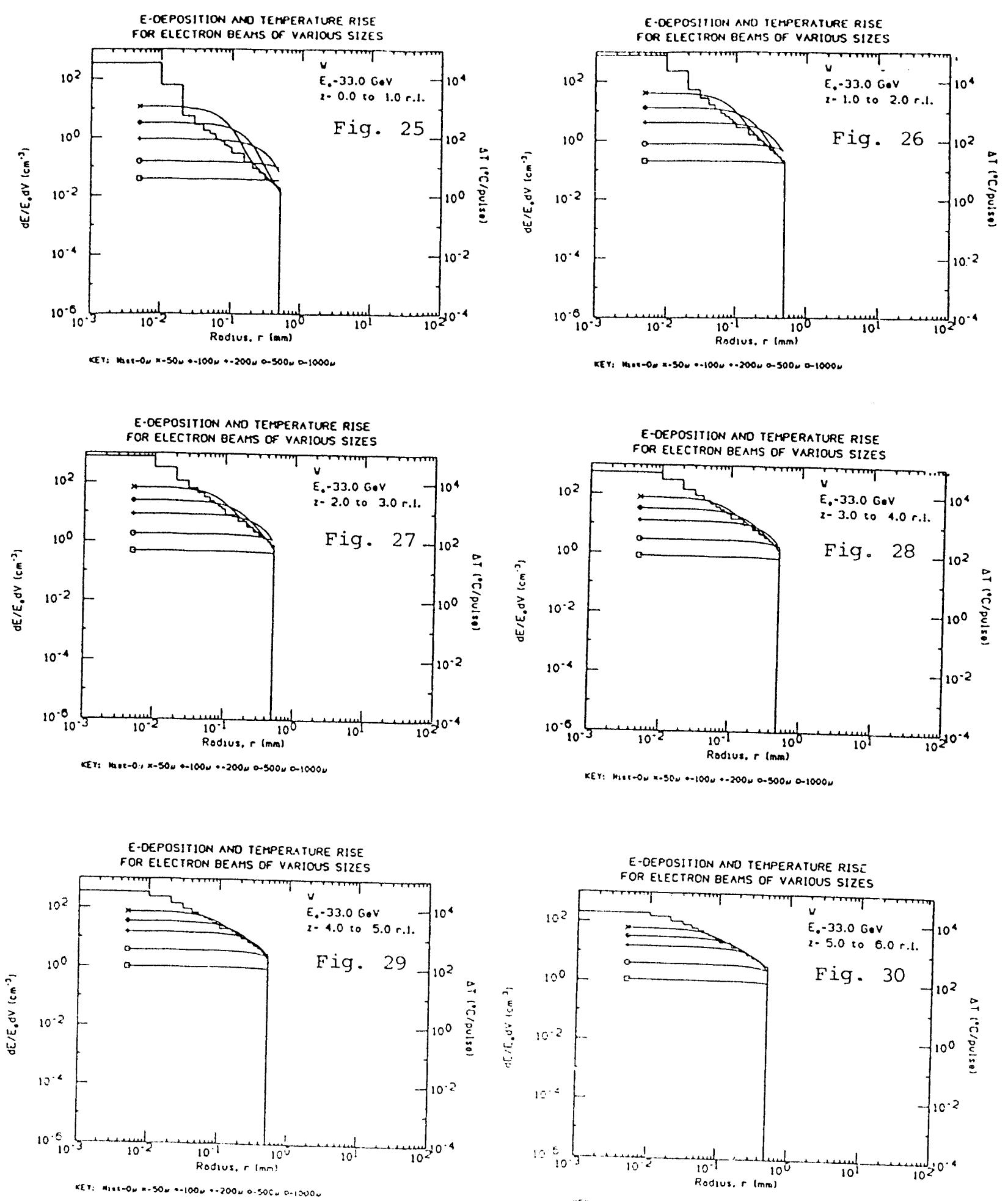

Figs. 25 30. Energy'deposition density and temperature-rise plots. 


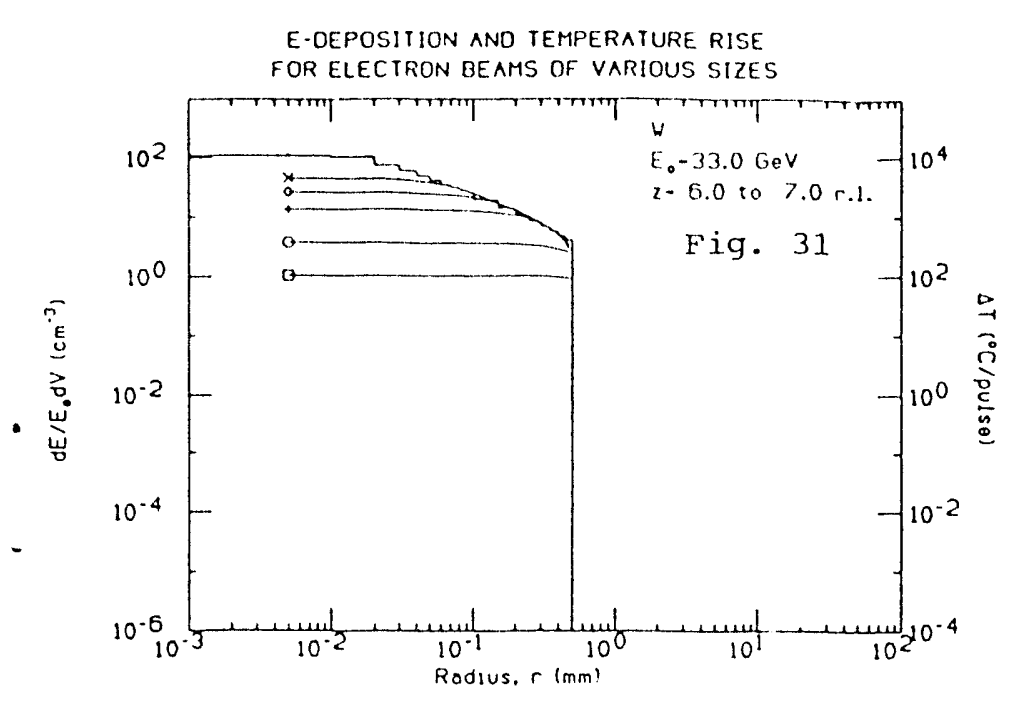

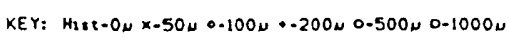

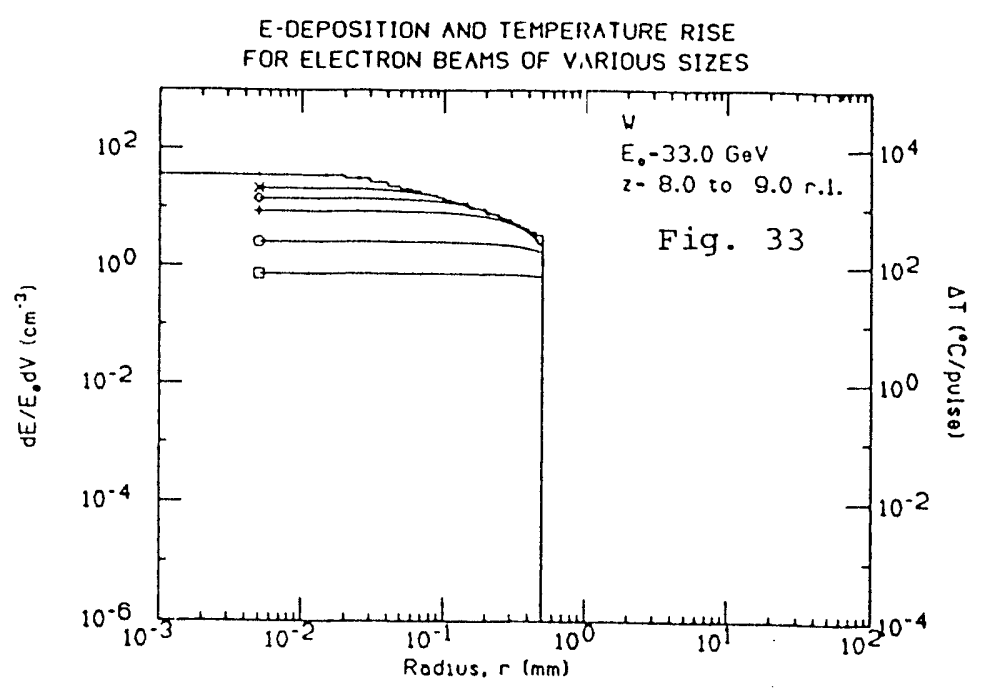

XEY: Het -0 N X-50N $0-100$ N +-200 N $0-500 N 0-1000$ N

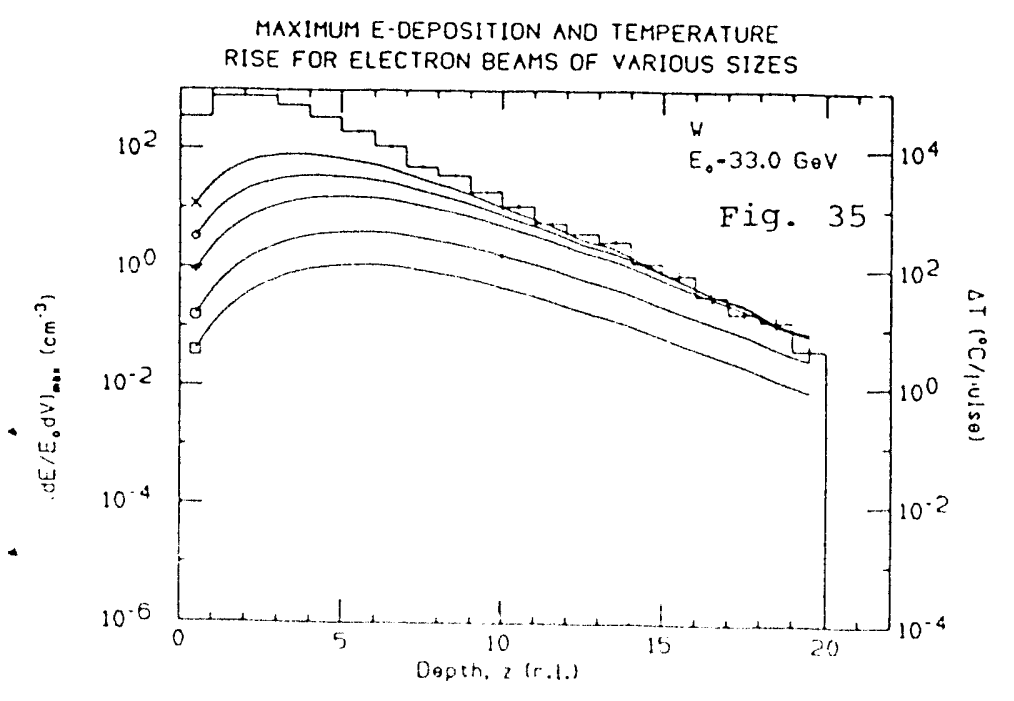

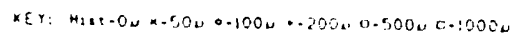

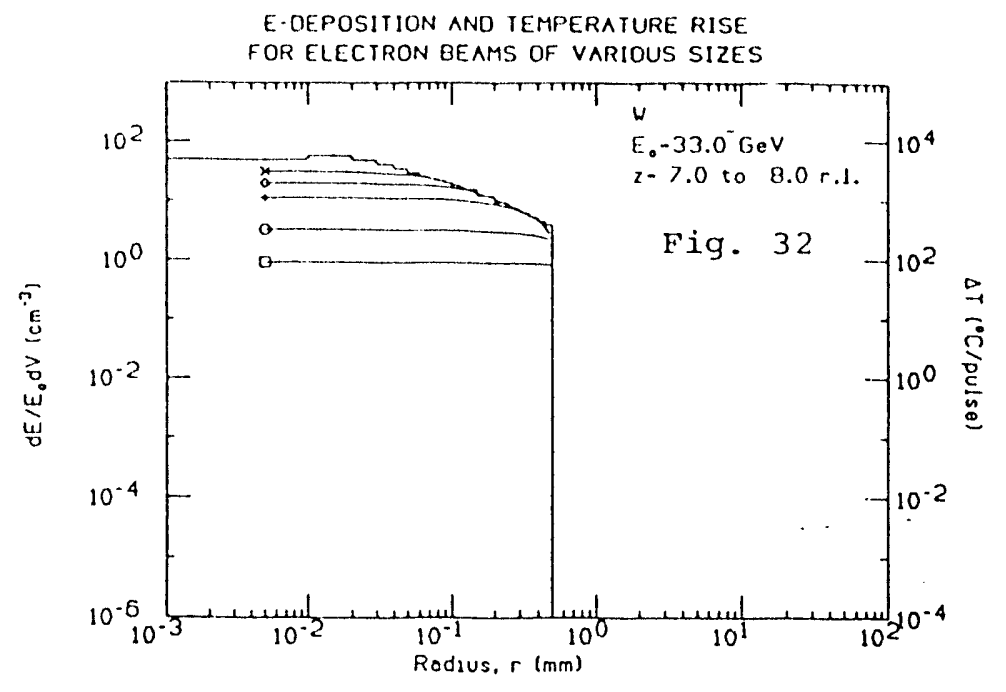

KEY: H3IT-ON $\times-50 N-100 N+-200 N 0-500 N$ 0-1000N

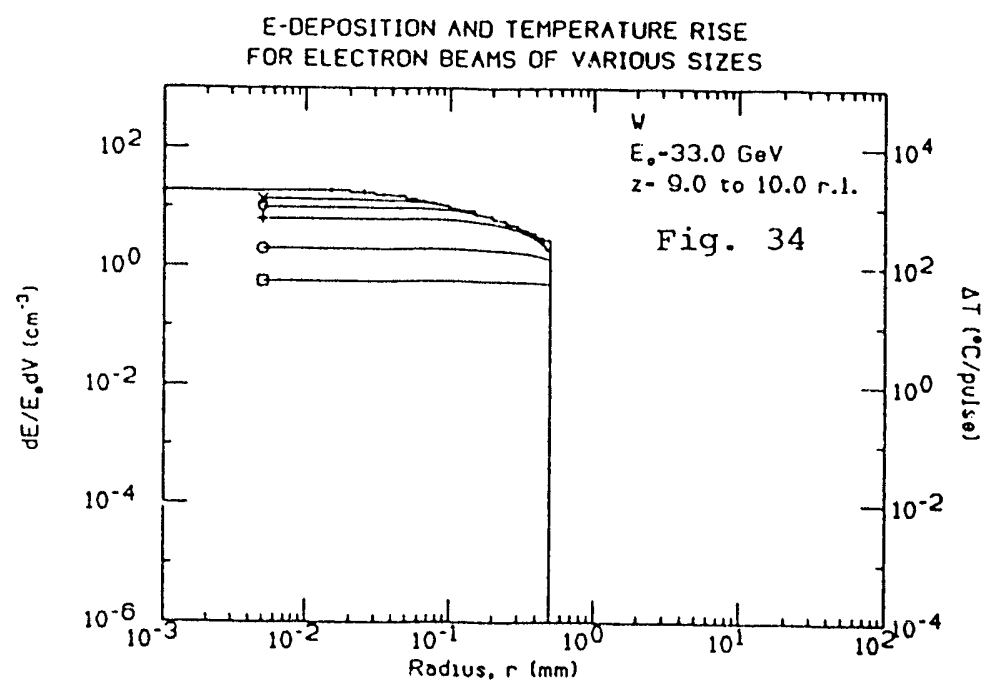

KEY: Het-0N X-50N $-100 \mathrm{~N} \cdot-.200 \mathrm{~N} 0-500 \mathrm{~N} 0.1000^{N}$

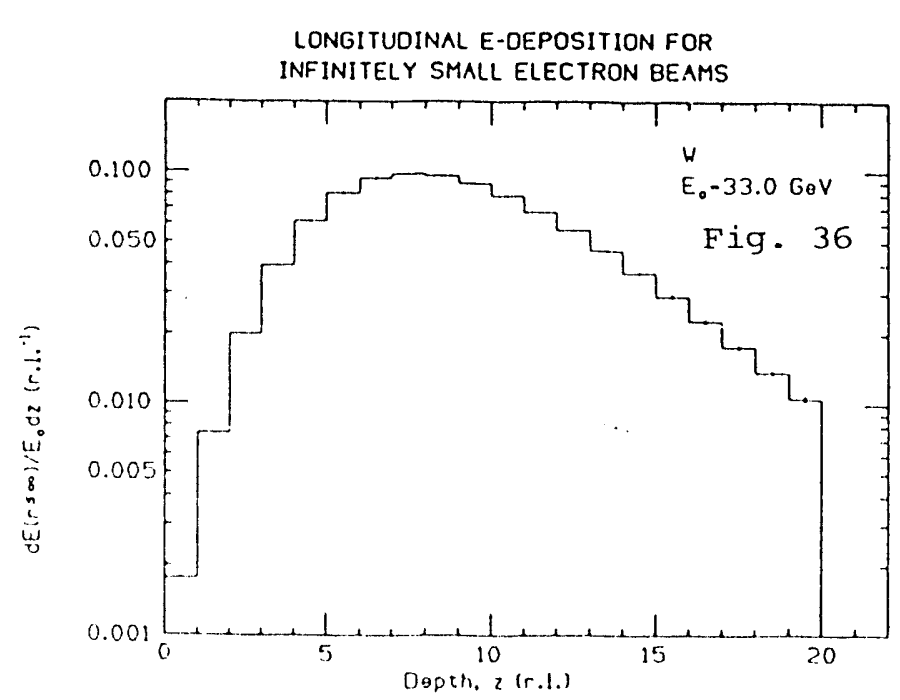

Figs. 31 36. Energy-deposition density and temperature-rise plois (and maximum and longitulina! sumarary plots). 


\subsection{Steady-State Temperature Rise}

The dominant mode of heat removal for a hot wire in vacuum will be radiant heat transport. The equation for a hot wire with cross-sectional area $A$ is given by $^{[39]}$

$$
q=\sigma A \epsilon\left(T_{\text {wire }}^{4}-T_{\text {wall }}^{4}\right) \quad(\text { Watts })
$$

where

$$
\begin{aligned}
& \sigma=\text { Stefan }- \text { Boltzmann constant }=5.669 \times 10^{-8}\left(\text { Watts } \mathrm{m}^{-2} \mathrm{~K}^{-4}\right) \\
& A=\text { surface area of wire }\left(\mathrm{m}^{2}\right) \\
& \epsilon=\text { emissivity. }
\end{aligned}
$$

The hottest part of the wire will be at the center, $r=0 . T_{\text {wire }}$ refers to the temperature on the outside surface of the wire. We are assuming that the temperature difference across a 0.5 - $\mathrm{mm}$ tungsten wire will be negligible. Therefore, $Z^{\prime}{ }_{\text {wire }} \approx T_{r=0}$. The current SLC incident electron beam is $33 \mathrm{GeV}$ and contains about $1 \times 10^{10} \mathrm{e}^{+}$per pulse at $120 \mathrm{~Hz}$. That is,

$$
1 \times 10^{10}\left(\frac{\mathrm{e}^{-}}{\text {pulse }}\right) \cdot 120\left(\frac{\text { pulses }}{\mathrm{sec}}\right) \cdot 33000\left(\frac{\mathrm{MeV}}{\mathrm{e}^{-}}\right) \cdot \frac{\mathrm{kw}-\mathrm{sec}}{6.243 \times 10^{15} \mathrm{MeV}}=6.3(\mathrm{kWatts})
$$

From EGS4 runs, the target region absorbs about $15 \%$ of the beam power. This corresponds to 950 Watts of absorbed power in the target, which corresponds to a wire temperature of

$$
\begin{aligned}
r_{\text {wire }}=\left\{\frac{q}{\sigma A \epsilon}\right\}^{\frac{1}{4}} & =\left\{\frac{950}{\left(5.669 \times 10^{-8} \cdot \frac{\text { Watts }}{\mathrm{m}^{2} \mathrm{~K}^{4}}\right) 2 \pi\left(0.5 \times 10^{-3} \mathrm{~m} \times 0.035 \mathrm{~m}\right)(0.39)}\right\}^{\frac{1}{4}} \\
& \simeq 4400{ }^{\circ} \mathrm{K} \simeq 4100^{\circ}(!
\end{aligned}
$$

Note that the melting point of tumgsten is $: 3400^{\circ}(\mathrm{!}$ 
If we take into account conduction by assuming that the wire is supported from a wall which is cooled and heat is conducted back through the wire, the equations become

$$
q=-k A_{x \operatorname{sect}} \frac{d T}{d x}+\sigma A_{s u r f a c e} \epsilon\left(T_{\text {uire }}^{4}-T_{\text {wall }}^{4}\right)
$$

or

$$
q=-k A_{x s e c t}\left(\frac{T_{\text {wire }}-T_{\text {wall }}}{l}\right)+\sigma A_{\text {surface }} \epsilon\left(T_{\text {wire }}^{4}-T_{\text {wall }}^{4}\right)
$$

where

1. $A_{x s e c t}=$ the cross-sectional area of the wire through which heat is conducted,

2. $A_{\text {surface }}=$ the surface area of the wire from which heat is radiated,

3. $l=$ the length of the wire.

Assuming that the wall temperature is zero (as a first approach), we have

$$
950=\frac{\left(1.2 \frac{\text { Watts }}{\mathrm{cm}^{\circ} \mathrm{K}}\right) \pi(0.05 \mathrm{~cm})^{2}}{3.5 \mathrm{~cm}} T+\left(5.669 \times 10^{-8} \frac{\text { Watts }}{\mathrm{m}^{2}{ }^{\circ} \mathrm{K}}\right)\left(1.1 \times 10^{-4} \mathrm{~m}^{2}\right)(0.39) T^{4}
$$

or

$$
950=2.69 \times 10^{-3} T+2.43 \times 10^{-12} T^{4} .
$$

This is solved by iteration as shown in Table 3 .

Table 3. Convergence by iteration.

\begin{tabular}{|c|c|c|c|c|}
\hline$q$ & Temp $\left({ }^{\circ} \mathrm{K}\right)$ & Conduction Term & Radiation Term & $q^{\prime}$ \\
\hline 950 & 3000 & 8.1 & 196.8 & 204.9 \\
950 & 4000 & 10.8 & 622.1 & 672.9 \\
950 & 4500 & 12.1 & 996.5 & 1009. \\
950 & 4300 & 11.6 & 830.8 & 842.4 \\
950 & 4400 & 11.8 & 910.8 & 92.3. \\
\hline
\end{tabular}


The solution converges at approximately the same temperature as beforei.e., when we assumed radiative losses only. Note that the conduction term only accounts for about $1 \%$ of the total heat loss. We can therefore conclude that conduction results in negligible cooling of the wire.

If we assume a reasonable wire temperature $\left(\sim 2000^{\circ} \mathrm{K}\right)$, we can find the maximum beam parameters. Assuming radiative losses only (since this represents $99 \%$ of the heat loss mechanism)

$$
q=\sigma A \epsilon T_{w i r e}^{4}=\left(5.669 \times 10^{-8} \frac{\text { Watts }}{\mathrm{m}^{2}{ }^{\circ} \mathrm{K}^{4}}\right)\left(1.1 \times 10^{-4} \mathrm{~m}^{2}\right)\left(2000^{\circ} \mathrm{K}\right)^{4}
$$

$$
\simeq 100 \text { Watts }
$$

In terms of $33 \mathrm{GeV}$ electrons

$$
0.1 \mathrm{kWatts} \cdot 6.243 \times 10^{15} \frac{\mathrm{MeV}}{\mathrm{kWatt}-\mathrm{sec}} \cdot \frac{\mathrm{e}^{-}}{33 \times 10^{3} \mathrm{MeV}}=1.9 \times 10^{10} \frac{\mathrm{e}^{-}}{\mathrm{sec}},
$$

which is about $1.6 \times 10^{8} \frac{\mathrm{e}^{-}}{\text {pulse }}$ at $120 \mathrm{~Hz}$.

If the current target gives about $2.7 \frac{e^{+}}{e^{-}}$yield ${ }^{(34)}$, this corresponds to a yield of $2.7 \times 10^{10} \frac{e^{+}}{p u l s e}$. The wire target yield is $6.8 \frac{e^{+}}{e^{-}}$. This corresponds to $1.1 \times 10^{9} \frac{e^{+}}{p u l s e}$. Barring a more efficient method of heat removal, the wire target yield is down by a factor of 25 from the current Sl, target.

One way to optimize the target is to vary the radius and see what happens to the temperature-i.e., sacrifice a little $\mathrm{e}^{+} / \mathrm{e}^{-}$yield for a cooler wire temperature. Assuming that radiative heat transfer is the only important mode for heat renoval, 
we know that

$$
\frac{q}{A} \propto T_{\text {wire }}^{4}
$$

or

$$
\frac{q}{r^{2}} \propto T_{\text {wire }}^{4}
$$

We also know that $q$ is proportional to the incident beam power (which is fixed) multiplied by the fraction absorbed in the wire, $f$, which varies with the radius. We can therefore rewrite the above equation as

$$
T_{\text {wire }} \propto\left\{\frac{f(r)}{r^{2}}\right\}^{\frac{1}{4}}
$$

To find $f(r)$, five separate EGS4 jobs were run and the energy absorbed in the target region was tallied. All runs assumed $33 \mathrm{GeV}$ electrons incident on a 10-r.l. $(3.5-\mathrm{cm})$ tungsten wire of varying radii. The output is shown in Table 4 and plotted in Fig. 37.

Table 4. Energy absorbed in target vs. target radius.

\begin{tabular}{|c|c|}
\hline Target Radius (mm) & $\begin{array}{c}\text { \% Energy Absorbed } \\
\text { In Target }(f)\end{array}$ \\
\hline 0.1 & 3.6 \\
0.5 & 15.2 \\
1.0 & 25.2 \\
5.0 & 50.1 \\
$\infty$ & 54.2 \\
\hline
\end{tabular}




\section{Energy Deposition Fract on in Target}

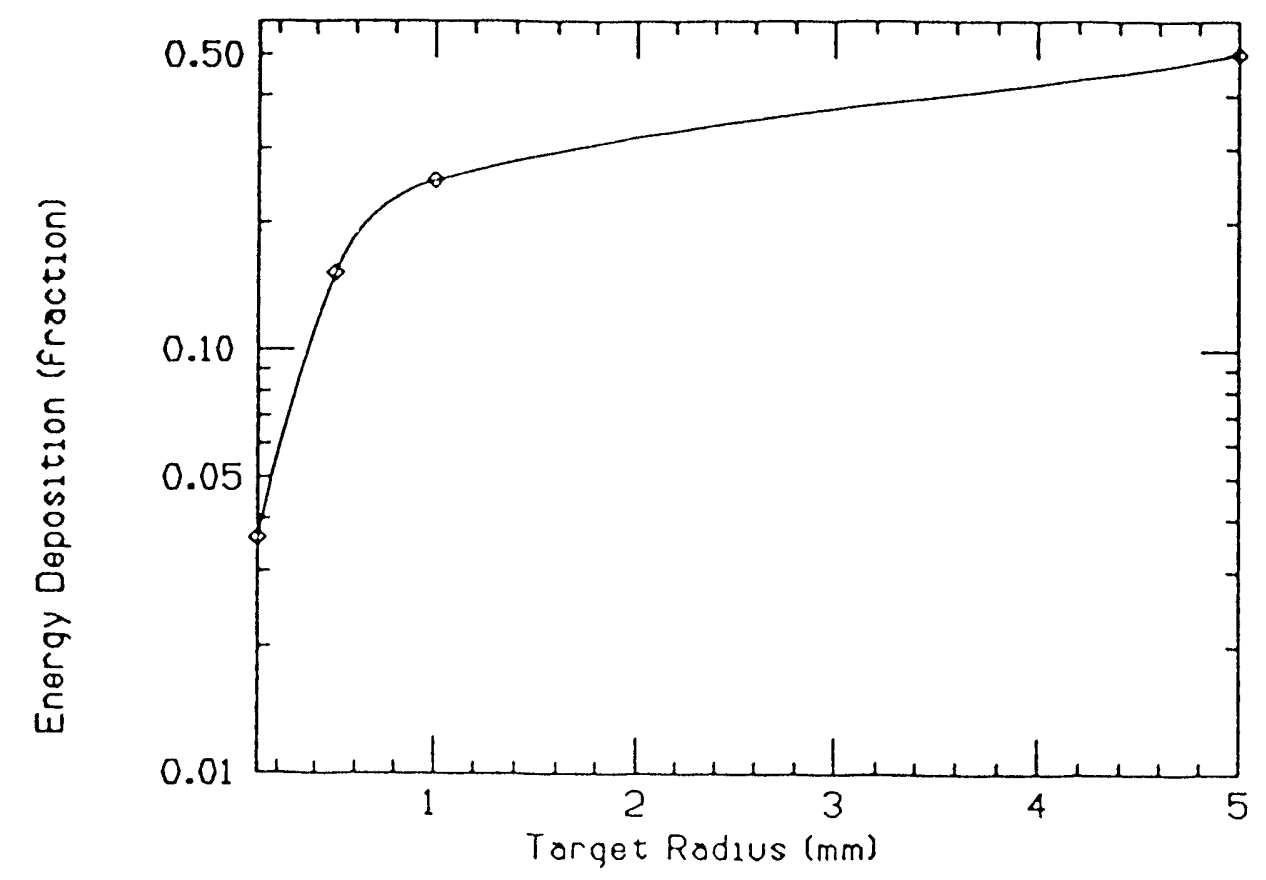

Fig. 37. Energy Deposition Fraction in Target.

Fitting this shape to a $3^{\text {rd }}$ order polynomial we get

$$
f(r)=1.27 \times 10^{-2}+0.28 r-3.65 \times 10^{-2} r^{2}
$$

Substituting this expression back into the previous equation, we get

$$
T_{u i r e}=\left\{\frac{0.28 r-3.65 \times 10^{-2} r^{2}}{r^{2}}\right\}^{\frac{1}{4}}
$$

We know that with a target radius of $0.0 \mathrm{i}-\mathrm{cm}$ (the point of optimum $\frac{e^{+}}{e-}$ ! ield), the wire reaches a stearly-state temperature of $\simeq 4100^{\circ}$ (; neglecting the melting point. We can now rary the above expression as shown in Table 5. 
Table 5. Target radius vs. temperature.

\begin{tabular}{|c|c|c|c|}
\hline Target Radius $(\mathrm{mm})$ & R.H.S. & Normalized R.H.S. & $\mathrm{T}\left({ }^{\circ} \mathrm{C}\right)$ \\
\hline 0.1 & 2.764 & 3.25 & 13000 \\
0.5 & 0.821 & 1.0 & 4100 \\
1.0 & 0.702 & 0.83 & 3400 \\
5.0 & 0.374 & 0.44 & 1800 \\
25.0 & 0.180 & 0.20 & 820 \\
\hline
\end{tabular}

These results are plotted in Fig. 38 below. One note of caution: as the radius increases, conduction becomes more important and the assumption of uniform heating is not valid. Therefore, these results are not valid for radii greater than a few times $\sigma_{b e a m}$, or a few millimeters.

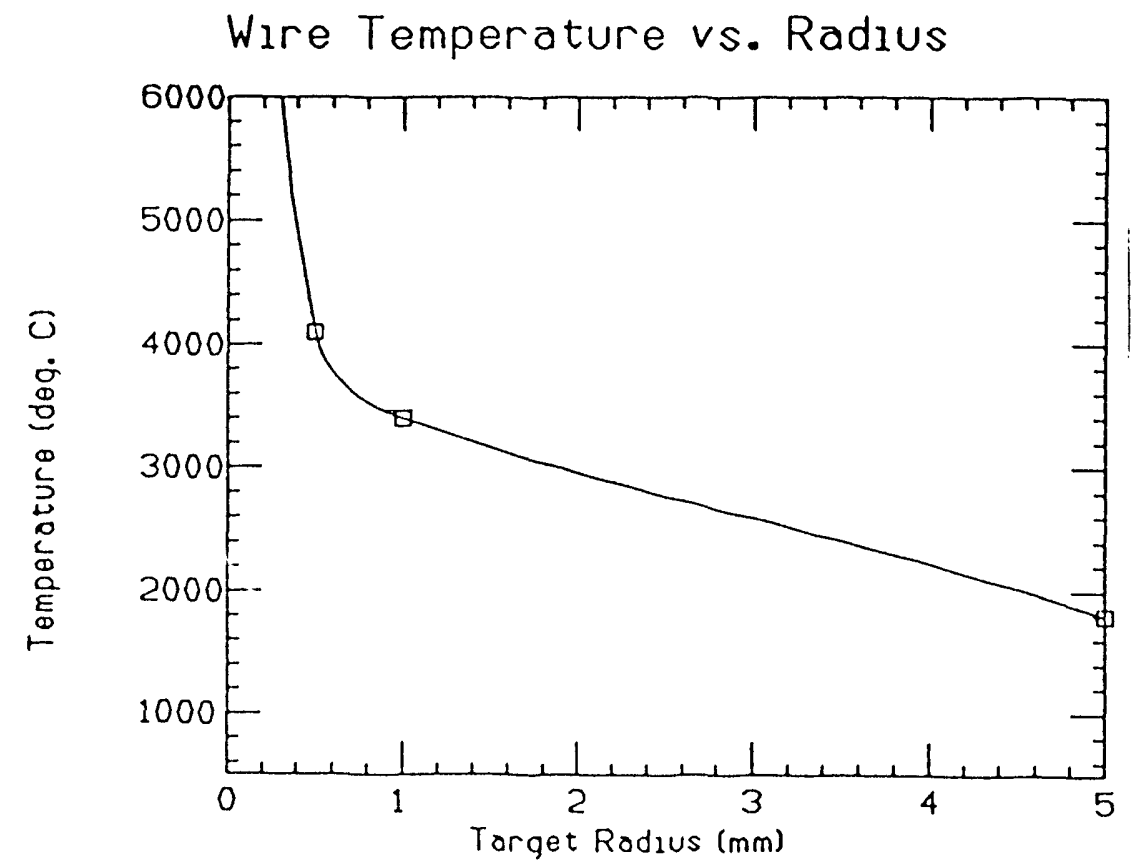

Fig. 38. Target Radius vs. Temperature.

From a previous figure showing relative yields vs. target radius (Fig. 16), we know that increasing the wire from $0.5-\mathrm{mm}$ radius to $1.0-\mathrm{mm}$ radius only drops the yield by about $20 \%$. Increasing the radius much more and the yield begins to 
approach that of the current slab target. From the figure above we can see that increasing the wire target radius much beyond the proposed $0.5-\mathrm{mm}$ does not drop the temperature enough to make a difference.

From the analysis in this section, it appears that severe heating restrictions must be placed on the wire to prevent melting. A better method of heat removal must be found in order to make the wire target feasible.

\section{Discussion}

It has been shown that the absolute yield $\left(\frac{e^{+}}{e^{-}}\right)$from the proposed wire target is a factor of 2.5 higher than the current target using the present SLC capture system, and is a factor of 3.4 higher if an enhanced capture system is employed (see Table 1). It has also been shown that the wire target is severely restricted by heat-loss mechanisms when compared to the current target.

To prevent the wire from melting, for example, the intensity of the incident electron beam must be reduced such that the wire yield is about a factor of 25 below the current target yield. There are several ideas which should be investigated in the future and may be used in parallel to enhance the wire target yield, perhaps enough to make it feasible:

- There are a large number of positrons created off the sides of the wire which are not captured by the present SI.C collector. It may be possible with the wire target to insert part of it into the flux concentrator. The large transverse dimensions of the current (slab) target, on the other hand, preclude doing this (and very few positrons escape the sides of this target anyways).

- Minute heat fins may be placed along the length of the wire to increase the conduction surface area. This woulia also add structural stability to the wire.

- The wire may be placed ontside the vacum system and some kind of conrective cooling nsed. 
- The wire could be surrounded by a large graphite region and then surrounded by copper which could be water cooled. This will eliminate radiative cooling but increase conductive cooling. However, Fig. 17 shows that the yield would be reduced by almost a factor of two. 


\section{REFERENCES}

1. R. Ruth, "Prospects, for Next-Generation $\mathrm{e}^{+} \mathrm{e}^{-}$Colliders", SLAC-PUB-4778 (1988).

2. R. Chehab, "Positron Sources", Laboratoire de l'Accelerateur Lineaire, LAL-RT-89-02 (1989).

3. B Rossi, High-Energy Particles (Prentice-Hall, 1952).

4. W. R. Nelson, H. Hirayama and D. W. O. Rogers, "The EGS4 Code System", SLAC-265 (1985).

5. Y. S. Tsai, "Pair Production and Bremsstrahlung of Charged Leptons", Rev. Mod. Phy. 46 (4) (1974) 815.

6. M. Aguilar-Benitez (Particle Data Group), "Review of Particle Properties", Physics Lett. B239 (1990) III.14.

7. E. Longo and I. Sestili, "Monte Carlo Calculation of Photon-Initiated Electromagnetic Showers in Lead Glass" Nucl. Instr. Meth. 128 (1975) 283.

8. Private communication with A. Kulikov, SLC Accelerator Physics Group (1990).

9. E. Freytag, Strahlensch?tz an Hochenergiebeschleunigern (G. Braun, Karlsruhe, 1972).

10. R. D. Evans, The Atomic Nucleus (McGraw-Hill, 19.55).

11. K. R. Kase and W. R. Nelson, Concepts of Radiation Dosimetry (Pergamon Press, 1978).

12. M. J. Berger and S. M. Seltzer, "Tables of Energy Losses and Ranges of Electrons and Positrons", NASA-SP-3012 (156(4).

13. N. F. Mott, Proc. Roy. Soc. (London) A 124 (1929) 425); A 135 (1932) 429. 
14. R. L. Ford and W. R. Nelson, "The EGS Code System: Computer Programs for the Monte Carlo Simulation of Electromagnetic Cascade Showers (Version 3)", SLAC-210 (1978).

15. A. J. Cook, "Mortran3 Users Guide", SLAC Computation Research Group Technical Memorandum Number CGTM 209 (1983).

16. E. A. Straker, P. N. Stevens, D. C. Irving and V. R. Cain, "MORSE-CG, General Purpose Monte-Carlo Multigroup Neutron and Gamma-Ray Transport Code With Combinatorial Geometry", Radiation Shielding Information Center (ORNL) Report CCC-203 (1976).

17. Y. Namito, T. M. Jenkins and W. R. Nelson, "Viewing MORSE-CG Radiation Transport With 3-D Color Graphics", SLAC-PUB-5170 (1990).

18. R. Beach, "The Unified Graphics System", SLAC Computation Research Group Technical Memorandom Number CGTM 203 (1985).

19. R. Cowan, "Use of 3-D Color Graphics with EGS", Comp. Phys. Comm. 45 (1987) 485 .

20. R. Donahue and W. R. Nelson, "Distribution of Induced Activity in Tungsten Targets", SLAC-PUB-4728 (1988).

21. R. F. Covian and W. R. Nelson "Producing EGS4 Shower Displays With the Unified Graphics System", SLAC-TN-87-3 (1990).

22. T. M. Jenkins, W. R. Nelson and A. Rindi, Monte Carlo Transport of Electrons and Photons (Plenum Press, 1988).

23. K. R. Shortt, C. K. Ross, A. F. Bielajew and D. W. O. Rogers. "Electron Beam Dose Distributions Near Standard Inhomogeneities", Phys. Med. Biol. 31 (1986) 235 .

24. Bruce A. Faddegon, "Bremsstrahlung of 10 to $30 \mathrm{MeV}$ Electrons Incident on Thick Targets". Ph.D. Thesis, Department of Physics, Carleton University' $(1990)$. 
25. H. L. Lynch, "Documentation of E'TRANS Program", memofrom H. L. Lynch to SLC Positron Source Group (27 October 1988).

26. F. Bulos et al., "Design of a High Yield Positron Source", SLAC-PUB-3635 (1985).

27. J. E. Clendenin, "High Yield Positron Systems for Linear Colliders", SLACPUB-4743 (1989).

28. J. E. Clendenin et al., "SLC Positron Source Startup", SLAC-PUB-4704 (1988).

29. S. Ecklund, "Positrons for Linear Colliders", SLAC-PUB-4484 (19S7).

30. J. E. Clendenin, S. D. Ecklund and H. A. Hoag, "The High-Gradient SBand Linac for Initial Acceleration of the SLC Intense Positron Binch", SLAC-PUB-5049 (1989).

31. A. F. Bielajew, D. W. O. Rogers, "PRESTA - The Parəmeter Reduced Electron-Step Transport Algorithm", Nucl. Instr. Meth B18 (1987) 165.

32. A. F. Bielajew, R. Mohan and C. Chui, "Improved Bremsstrahlung Photon Angular Sampling in the EGS4 Code System", NRCC-PIRS-0203 (1989).

33. H. W. Koch and J. W. Motz, "Bremsstrahlung Cross-section Formulas and Related Data", Rev. Mod. Phys. 31(4) (1959) 920.

34. M. James, R. J. Donahue, R. Miller and W. R. Nelson, "A New Target Design and Capture Strategy for High-Yield Positron Production in Electron Linear Colliders", SLAC-PUB-5215 (1990).

35. H. DeStaebler, "Temperature Calculations for the Positron Target", SLC. Single Pass Collider Memo CN-21 (1980).

36. H. DeStaebler, "Calculations for Positron Target 'lest in ESA" SLC Single Pass Collider Memo (N .2:3 (19S0).

37. H. DeStaebler, "More Calculations for Positron Target 'Test in ESA" SLC" Single Pass Collider Memo ( N 24 (1980). 
38. S. Ecklund and W. R. Nelson, "Energy Deposition and Thermal Heating in Materials Due to Low Emittance Electron Beams", SLC Single Pass Collider Memo CN-135 (1981).

39. J. P. Holman, Heat Transfer (McGraw-Hill, 1986). 


\section{ACKNOWLEDGMENTS}

I would like to express my thanks to Dr. Ralph Nelson of the Radiation Physics Group at the Stanford Linear Accelerator Center who has acted as an on-site advisor to this work. It was his imagination and hard work which led to this project. I wish to thank him for the many days (and nights) he put aside for me in his busy schedule. He has given me much encouragement and serves as a source of inspiration.

I also wish to express my thanks to Dr. Jose Martin of the University of Lowell for further improving the work. He has helped make this effort possible across three thousand miles. 

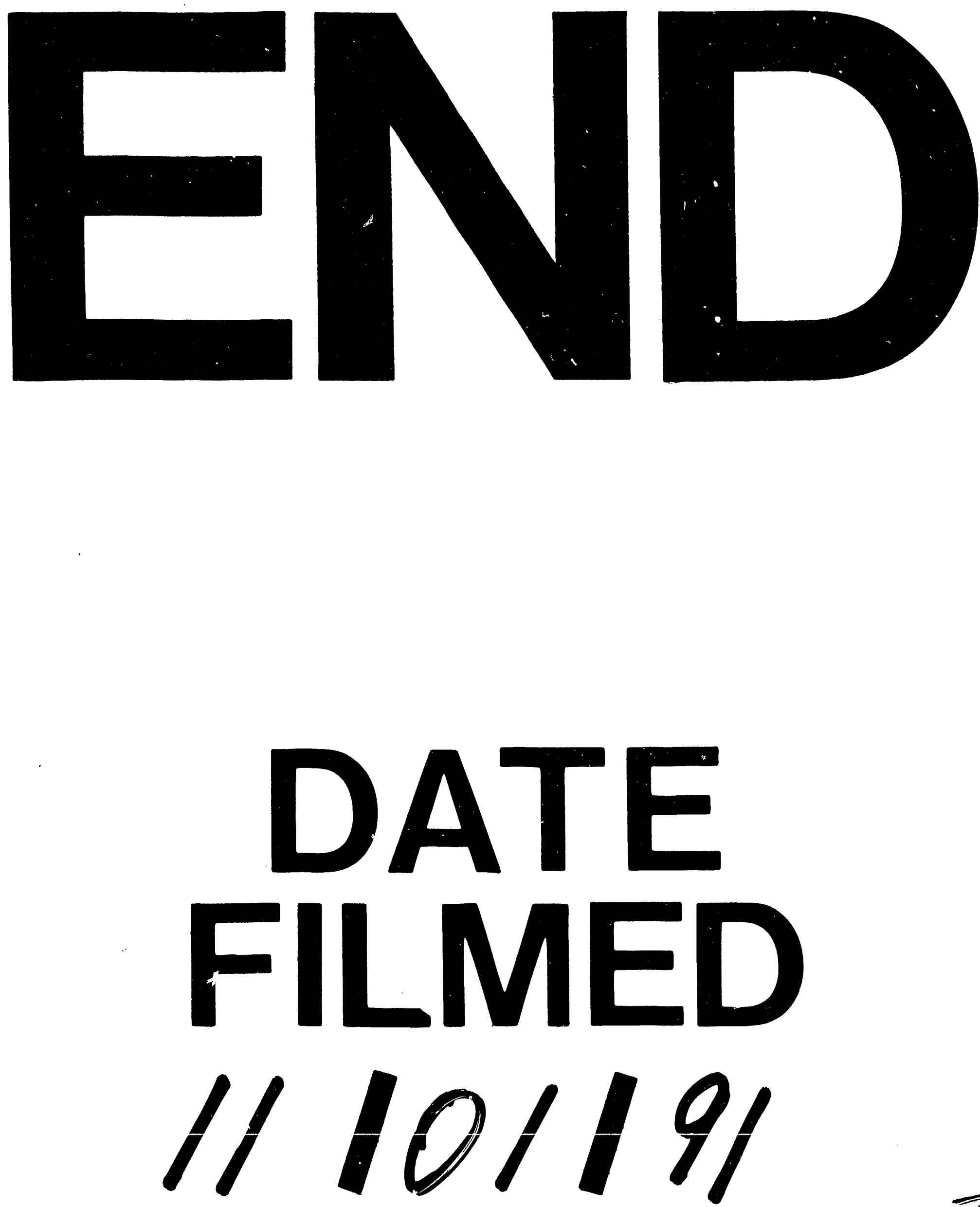